\title{
ROMY: A Multi-Component Ring Laser for Geodesy and Geophysics
}

Heiner Igel ${ }^{1}$, K. Ulrich Schreiber ${ }^{2}$, André Gebauer ${ }^{1,2}$, Felix Bernauer ${ }^{1}$, Sven Egdorf ${ }^{1}$, Andrea Simonelli ${ }^{1,3}$, Chin-Jen Lin $^{1,4}$, Joachim Wassermann ${ }^{1}$, Stefanie Donner ${ }^{1,8}$, Céline Hadziioannou ${ }^{1,5}$, Shihao Yuan ${ }^{1}$, Andreas Brotzer ${ }^{1}$, Jan Kodet ${ }^{2}$, Toshiro Tanimoto ${ }^{6}$, Urs Hugentobler ${ }^{2}$, and Jon-Paul R. Wells ${ }^{7}$

${ }^{1}$ Ludwig-Maximilians-University Munich, Germany

${ }^{2}$ Fundamentalstation Wettzell, Technical University Munich, Germany

${ }^{3}$ now at INFN, Pisa, Italy

${ }^{4}$ now at Academia Sinica, Taipeh, Taiwan

${ }^{5}$ now at University of Hamburg, Germany

${ }^{6}$ University of Santa Barbara, California

${ }^{7}$ School of Physical and Chemical Sciences, University of Canterbury, and Dodd-Walls

Centre for Photonic and Quantum Technologies, Christchurch, New Zealand

${ }^{8}$ now at Bundesanstalt für Geowissenschaften, Hannover, Germany 


\section{In original form 2020 October 5}

Corresponding author: Heiner Igel, heiner.igel@1mu.de

This manuscript is a preprint and has been submitted for publication in the Geophysical Journal International. It has not undergone peer review yet. Subsequent versions of this manuscript may have slightly different content. If accepted, the final version of this manuscript will be available via the 'Peer-reviewed Publication DOI' link on the right - hand side of this webpage. Please feel free to contact the corresponding author. We welcome feedback.

Coauthor emails:

Schreiber, Ulrich <ulrich.schreiber@tum.de>

Felix Bernauer < fbernauer@geophysik.uni-muenchen.de>

Sven Egdorf <egdorf@geophysik.uni-muenchen.de>

Andrea Simonelli <andrea.simonelli.as@gmail.com>

Chin Jen Lin <youngman@earth.sinica.edu.tw>

Joachim Wassermann <joachim.wassermann@geophysik.uni-muenchen.de>

Stefanie Donner <stefanie.donner@bgr.de>

Celine Hadziioannou <celine.hadziioannou@uni-hamburg.de>

Shihao Yuan <syuan@geophysik.uni-muenchen.de>

Andreas Brotzer <brotzer@geophysik.uni-muenchen.de> Kodet, Jan <jan.kodet@ tum.de>

Toshiro Tanimoto <toshirotanimoto@ucsb.edu>

Hugentobler, Urs <urs.hugentobler@tum.de>

Jon-Paul Wells <jon-paul.wells@ canterbury.ac.nz>

André Gebauer <gebauer@fs.wettzell.de> 


\section{Abstract}

Single-component ring lasers have provided high-resolution observations of Earth's rotation rate as well as local earthquake- or otherwise-induced rotational ground motions. Here we present the design, construction, and operational aspects of ROMY, a four-component, tetrahedral-shaped ring laser installed at the Geophysical Observatory Fürstenfeldbruck near Munich, Germany. Four equilateral, triangular-shaped ring lasers with 12 m side length provide rotational motions that can be combined to construct the complete vector of Earth's rotation from a point measurement with very high resolution. Combined with a classic broadband seismometer we obtain the most accurate 6 degree-of-freedom ground motion measurement system to date, enabling local and teleseismic observations as well as the analysis of ocean-generated Love and Rayleigh waves. The specific design and construction details are discussed as are the resulting consequences for permanent observations. We present seismic observations of local, regional, and global earthquakes as well as seasonal variations of oceangenerated rotation noise. The current resolution of polar motion is discussed and strategies how to further improve long-term stability of the multi-component ring-laser system are presented.

Keywords: Earth's rotation; ring laser; rotational seismology.

\section{Introduction}

Sensing rotational motions in general has a wide range of applications, reaching from the control of robotic movements, navigation tasks in flight and space operations, to measuring Earth's and planetary rotation, rotational ground motions due to earthquakes, and vibrations of buildings. Optical Sagnac interferometers such as passive fibre-optic gyros or active ring laser gyros outperform mechanical devices by orders of magnitude and are the technical choice for high-resolution, broadband observations of rotational motions in geodesy and 
geophysics (Schreiber et al., 2014). Output of the ring laser is the beat frequency of two counter-propagating laser beams (e.g., Schreiber et al., 2006b) that is directly proportional to the rotation rate perpendicular to the plane of the laser beams.

An extremely sensitive ring laser system (G-ring) was installed in 2002 at the Geodetic Observatory Wettzell (Schreiber et al., 2009c) measuring the local component of rotation around the vertical axis. The G-ring was specifically designed for geodesy, built on a monolithic Zerodur structure, buried underground, thus providing sufficient long-term stability to be able to resolve tidal effects and polar motion (e.g., Schreiber et al., 2003, 2011). As with many observation systems one person's noise is another person's signal. The G-ring observations of Earth's rotation are superimposed by local rotational ground motions from a variety of sources. The unprecedented high-resolution (single-component) ground rotational observations of the Gring of local, regional, and teleseismic earthquakes (Igel et al., 2005; Cochard et al., 2006; Igel et al., 2007) triggered research into the potential of using additional rotation components for seismological research questions. The observation of rotations was already promoted by theoretical seismologists like Aki and Richards (2002) for a number of reasons, clearly pointing out that there is a lack of sensors recording this type of ground motion. Many developments of this new field (rotational seismology) in terms of instrumentation, theory, and applications have been documented in recent review articles (Schmelzbach et al., 2018; Igel et al., 2015; Li and van der Baan, 2017) and two special issues (Lee et al., 2009; Igel et al., 2012). From an instrumentation point of view these developments can be subdivided into two categories: 1) the high-resolution observatory-style recording systems like ring lasers as discussed in this paper and 2) portable rotation sensors that only recently are considered fit for the specific requirements of seismic ground observations (e.g., Bernauer et al., 2012, 2018; Yuan et al., 2020b; Wassermann et al., 2020).

On the high-resolution side, ring lasers were identified to potentially improve and contribute to the observation of Earth's free oscillations (Widmer-Schnidrig and Zuern, 2009), where tilt-displacement coupling can 
substantially deteriorate classic seismometer observations. Indeed, toroidal free oscillations could be observed on the G-ring following large earthquakes (e.g., Igel et al., 2011; Nader et al., 2012). Also, the waveform match between rotational and translational ground motions of SH type motions (assuming plane waves) can be exploited to estimate propagation velocities (e.g., Igel et al., 2005; Cochard et al., 2006; Igel et al., 2007). This has considerable potential as a one-station method to determine local velocity structure (e.g., Edme and Yuan, 2016; Wassermann et al., 2016; Keil et al., 2020). Ring laser observations also contribute to the discussions on the origin of the ocean generated seismic noise. Due to the polarization-filter characteristics of rotation observations pure Love waves can be observed on vertical component rotation systems like the G-ring allowing precise characterization of time-dependent Love-to-Rayleigh energy ratios in the microseismic band (e.g., Tanimoto et al., 2015, 2016).

More relevant for field-type seismic experiments are the recent developments providing seismology with portable broadband rotation sensor technology (e.g., Bernauer et al., 2012, 2018; Brokesova et al., 2012; Jaroszewicz et al., 2012). With appropriate sensitivity there is a broad spectrum of applications ranging from tilt-corrections to improve the quality of classic seismometer records (Lindner et al., 2017; Bernauer et al., 2020), to site-effect characterization (e.g., Keil et al., 2020), seismic source inversion (e.g., Donner et al., 2016), separation of wavefields (e.g., Sollberger et al., 2018), volcano seismology (e.g., Wassermann et al., 2020), seismic exploration (e.g., Li and van der Baan, 2017), or structural engineering (e.g., Trifunac, 2009; Schreiber et al., 2009d). The current portable rotation sensing technology is not sensitive enough to measure below the physical noise level of our planet (e.g., ocean generated noise). However, observatory-type ring-laser technology may provide the required sensitivity.

Finally, ring lasers allow the most accurate ground-based measurement of Earth's rotation. A singlecomponent horizontally-aligned ring laser, such as the G-ring, provides only a scalar quantity of the rotational component around the local vertical axis projected onto the axis of Earth's rotation. This motivates the devel- 
opment of an (at least) three-component ring laser sensor that allows for the recovery of the complete vector of Earth's rotation. In addition to directly measuring changes in Earth's rotation rate and polar motion (e.g., Schreiber et al., 2004), it has been argued that ring laser measurements of the complete rotation vector ideally complement classic VLBI (Very Long Baseline Interferometry) observations (e.g., Mendes Cerveira et al., 2009; Gebauer et al., 2020).

The substantial interest in high-resolution rotation sensing both in geophysics and geodesy motivated the proposal to build a large multi-component ring laser system that serves both research fields. Here, we describe the four-component ring laser ROMY installed in the Geophysical Observatory Fürstenfeldbruck, Germany, in its final configuration and present first observations.

The paper is structured as follows. We 1) first discuss the various aspects that went into the final technical design of the ring laser instrument. This will be followed by 2) a brief description of the construction phase, 3) the operational principles, and 4) data analysis and a first review of the type of observations we obtain. This involves the observation of the complete vector of Earth's rotation as a function of time, high-resolution rotational ground motion due to earthquakes, and ocean generated seismic noise.

\section{ROMY: Design Considerations}

The main goal of the ROMY project was to build on the successful G-ring (e.g., Schreiber et al., 2006a), and GEOSENSOR (e.g., Schreiber et al., 2009b) concepts for both geodesy and seismology and to develop a multicomponent ring laser system with higher sensitivity for each component. There are a number of design aspects that have a strong impact on the final performance of the instrument. The detection sensitivity for a rotational signal strongly depends on the size of the ring cavity, on the actual linewidth of the laser radiation in the cavity, as well as on the overall geometrical sensor stability Pritsch et al. (2007). Therefore we had to maximize the ratio of the enclosed area $(\mathrm{A})$ over the perimeter $(\mathrm{P})$ of the gyro, whilst maintaining the highest possible 
mechanical stability at the same time. Although not related to the aspect of sensitivity, we also required a stable spatial orientation of at least 3 ring laser interferometers, each in a linear independent plane with respect to each other. Therefore we have chosen a tetrahedral design, which offered the additional opportunity to add a 4th redundant interferometer in order to achieve a better control over the consistency of the ROMY performance. By placing the apex of the tetrahedron at the bottom, we have considerably reduced the required excavation at the construction site. The stability of ROMY is also inherently high, as the scale factor defining corner points of all four rings are closely spaced together on a massive concrete foundation. With all these aspects working in our favor, we nevertheless had to make a compromise for the sensor sensitivity.

Very large ring lasers have predominantly been designed as squares or rectangles (Dunn et al., 2002; Hurst et al., 2004) in order to optimize their sensitivity. Since we have to maximize the ratio of A/P and at the same time to minimize the line broadening losses in the cavity, the best performance of a gyro is obtained by optimizing the worth function $\gamma=\frac{A}{P \cdot n}$, where $\mathrm{n}$ is the number of loss incurring mirrors in the cavity. For our role model gyro " $G$ " this worth function yields $\gamma=0.25$ and it was a design criterion to make each ring of ROMY at least twice as good, which led to the design length of each side of ROMY of $12 \mathrm{~m}$, providing a value of $\gamma=0.58$, assuming that the losses induced by the mirrors are comparable in both types of gyros, which is a reasonable expectation.

The final ring laser geometry is illustrated in Fig. 1a. Three sub-horizontal triangular ring lasers are oriented such as to maximize the normal vector projection with respect to Earth's rotation (see section on ROMY construction for details). The tetrahedron is inverted with the tip pointing vertically down. Each triangular ring laser has its own independent cavity enclosed by a vacuum recipient, a laser gain section and a data acquisition system. Each corner as well as the tip of the tetrahedron (at the bottom of the structure) can be accessed through a circular vault for installation and maintenance. These corners are illustrated through technical drawings in Fig. 1b-d. The laser radiation is accessible by the light leakage through the mirrors at 
each corner exiting through a viewport at the back of the vacuum enclosure. To establish a closed optical path an external alignment laser is injected into the cavity. All corner boxes can be rotated and tilted gently to obtain lasing. Note the bottom installation of the three corners, fixed to a rigid base plate attached to the concrete basement connected to the bedrock. This gives a rigid geometrical reference for the ring laser orientation.

The (temporal) stability and noise level of each ring laser component depends strongly on keeping the triangular geometry as rigid as possible. The G-ring (Schreiber et al., 2009a) is very stable because the entire body of the interferometer is built as a monolith from Zerodur, thermally almost a zero-expansion material. To apply the same design to ROMY would be prohibitively expensive, therefore we applied a heterolithic approach, where a solid concrete foundation provides the geometrical reference. One of the corner boxes in each ring laser component is adjustable by a piezo actuator in order to compensate thermal expansion. Utilizing an active control of the optical frequency in the cavity will eventually make ROMY a virtual monolithic structure.

In principle, three ring lasers would be sufficient to reconstruct the Earth's rotation vector and observe the complete rotational ground motion. While three sub-horizontal triangular cavities are enough to reconstruct the full Earth rotation vector, the final design included an additional interferometer in the horizontal plane, thus providing the vertical component of rotation additionally. This allows us to directly compare observations with the well established G-ring at a distance of around $200 \mathrm{~km}$. Note that due to limitations in the construction the circumference of the top ring laser component is slightly smaller than the sub-horizontal ones.

A tetrahedral shape with the tip pointing downwards leaves freedom for the orientation of the three subhorizontal faces. Ring lasers are active Sagnac interferometers. Earth rotation generates a beat note that biases all geophysical signals away from zero well outside the lock-in regime. Therefore the normal vector of each triangular plane should be as non-orthogonal to the Earth rotation vector as possible in order to make this bias value large. In ROMY this is realized by having one of the faces aligned with the N-direction, while the others 
point in the easterly and westerly direction respectively. This achieves nearly equal projections on the Earth rotation axis (see Fig. 1a).

\section{ROMY: Construction}

ROMY is a highly sensitive rotation sensor, which is operated in a strap-down configuration. This means that the ring laser structure has to be rigidly attached to the Earth's crust in order to guarantee that the recorded rotations in fact represent the ground motion. A design goal is the reliable detection of rotation rates of less than $1 \mathrm{prad} / \mathrm{s}$ in all three spatial directions. With an arm length of $12 \mathrm{~m}$ for each of the sides of the tetrahedron, this sets high requirements for the mechanical monument structure. At the same time it requires a careful procedure for the construction process itself, in order to ensure as little ground settling motions as possible. Furthermore, excavations had to be reduced to a bare minimum in order to maintain the overall terrain stability. In the first phase, the seamless integration of the concrete monument into the local terrain took place (Fig. 2a). This provided a rigid mounting platform for the beam lines of the laser interferometers. Since the scale factor of the gyros depend on the size of the enclosed area, the size had to be as stable and as large as possible. Therefore it was important to make the concrete support massive.

\subsection{Concrete Structure}

The design of the ring laser monument required further considerations. In order to reduce detrimental strain effects induced by wind friction (Gebauer et al., 2012) the top horizontal part of the concrete structure was required to be some $3 \mathrm{~m}$ underground. This also provides a better thermal isolation for an improved sensor stability.

Eventually the following procedure was adopted: 1) Excavate the required volume entirely, 2) secure the 
embankment with a shotcrete reinforcement, anchored to the surrounding terrain by long bolts, 3) construction

of a massive concrete structure from bottom to top rigidly supporting the inclined and horizontal beam lines of the laser cavities, and 4) adding a large circular access shaft at the center and smaller vaults at each top corner and halfway between them. This provides the necessary service access points for the alignment of the laser cavities and the gain sections of the laser excitation.

Due to the fact that the mirror supports are on two different floor levels, approximately $10 \mathrm{~m}$ apart in height and that the entire structure has to be stable to within a few wavelengths $(\approx 3 \mu \mathrm{m})$, it was required that the entire soil structure around the ROMY monument had to be left intact as far as possible. Removing and subsequently refilling large quantities of soil for a larger building structure would destabilize the terrain and gives rise to a subtle and continuous creep motion over many years until the soil has compacted again. For the installation of a laser interferometer this is clearly not adequate. Since the surrounding terrain was supported by a strong retaining wall during the excavation process, the creep of the terrain could be minimized. When the terrain was refilled after the integration of the monument, care was taken to properly compact the refill material. The construction phase took approximately six months. The final installation is illustrated in Fig. 2a and also shown in Hand (2017), supplemented by a video on youtube (https://youtu.be/MXYV6wNdZm8). This video also contains a compressed account of the entire construction phase.

\subsection{Ring Laser Components}

ROMY consists of four individual triangular ring cavities, within which the laser beams propagate. Three of these rings are tilted by about $57^{\circ}$ from the horizontal and there is an apex at the bottom of the monument, $14 \mathrm{~m}$ deep, where three corners are joined together (Fig. 2d). Angled granite support structures carry the mirror holder boxes (Fig. 2c), while the corner boxes for the horizontal ring are directly bolted to the concrete monument. The location of the corner boxes define the physical size of the structure and the corners are 
joined by stainless steel pipes to form the beam enclosure. Short bellows near the corner boxes (Fig. 2c) reduce deformations from strain and make sure that the mechanical rigidity of the corner construction is not compromised. In the middle of the uppermost side of each triangle, there is a $5 \mathrm{~mm}$ wide and $20 \mathrm{~cm}$ long capillary for laser excitation (Fig. 2b). The width of the capillary also acts as a spatial mode filter and has been designed to minimize the loss for the desired transverse TEM $_{0,0}$ laser mode. Higher-order transversal modes with a larger mode volume, however, are discouraged by increased loss. There are no additional Brewster windows or other loss increasing components anywhere inside the cavity. In fact, there are only the three curved super mirrors (radius-of-curvature $=12 \mathrm{~m}$ ) as interacting intra-cavity components with a specified total loss of approximately $12 \mathrm{ppm}$ per mirror (scatter, transmission and absorption) in the system.

Since the entire beam path is enclosed by an UHV (ultra-high vacuum) compatible enclosure (pipe and mirror box housing), the resonator can be evacuated and then filled with a mixture from $(0.2 \mathrm{hPa})$ neon and (6.3 hPa) helium. Lasing is achieved by radio frequency excitation. Figure 3 depicts the basic sensor concept. Due to the open gain section, the laser gas distributes all around inside the cavity. Overpressuring the laser cavity increases the homogeneous spectral linewidth of the cavity modes and thereby avoids mode competition (excitation of several neighboring transverse lasing modes) in the regime $\pm 90 \mathrm{MHz}$ around the lasing frequency. Therefore, it is possible to operate the interferometer on a single mode per sense of propagation, despite a longitudinal mode spacing below $9 \mathrm{MHz}$. Mode jumps for the laser are nonetheless not infrequent. The $36 \mathrm{~m}$ length of the laser cavity contracts or expands by up to $3 \mu \mathrm{m}$, provided by the increased line broadening. Another complication is the large internal stainless steel surface area of the vacuum recipient. Although each of the pipes was baked over several weeks to reduce the outgassing of hydrogen, the residual diffusion left in the cavity is still considerable. Contamination of the laser gas with hydrogen diminishes the achievable gain from the ${ }^{3} S_{2} \longrightarrow 2 P_{4}$ transition at $632.8 \mathrm{~nm}$ (red). The application of a CapaciTorr D 200 getter pump in each ring therefore reduces the effect of outgassing considerably (Schreiber and Wells, 2013), thus increasing 
the system stability. Currently it is possible to operate each of the four cavities for several months on a single gas fill.

In order to obtain a stable beat note, the laser beam power in the cavity has to be stabilized. A small portion of the light leaking through one of the mirrors is detected and amplified by a photo-multiplier. The resulting voltage is then fed back to drive the power of the radio frequency transmitter such that the laser radiation in the cavity remains constant. At another corner of the interferometer the two counter-propagating beams are taken out and superimposed via a beam combiner. The resultant beat note corresponds to the Sagnac frequency and is strictly proportional to the externally imposed rotation rate.

Figure 4 depicts an observed sample interferogram from the horizontal ring laser component. The fidelity of the measurement signal is well over $70 \mathrm{~dB}$ and the dynamic range may exceed 6 orders of magnitude. The challenge is to properly extract small variations of this frequency with sufficient stability over long observation times.

\section{ROMY: Principles of Operation}

The principle of ring lasers and the history have been well documented in recent review papers (e.g., Schreiber and Wells, 2013). We focus here on the essential aspects.

\subsection{Ring Laser Principle, Sagnac Effect}

A ring laser gyro constitutes a traveling wave oscillator, where two beams coexist, one traveling in the clockwise and one traveling in the counter clockwise direction. The effective length of the oscillator and hence its optical frequency depends on the rotation rate experienced by the cavity. When the cavity is at rest with respect to an inertial frame of reference the gyro is frequency degenerate and the beat note between the two counter- 
propagating waves disappears. However, when the gyro is rotated, the effective co-rotating cavity becomes slightly longer, while the anti-rotating cavity gets shorter by the same amount. The laser oscillation responds by adjusting the optical frequency of each sense of propagation to fit an integer number of wavelengths within the cavity, a necessary condition to satisfy laser coherent amplification. This means that the rotation rate experienced $(\dot{\Omega})$ around the normal vector $\mathbf{n}$ on the laser plane is strictly proportional to the frequency splitting $(\delta f)$ of the gyro:

$$
\delta f=\frac{4 A}{\lambda P} \mathbf{n} \cdot \dot{\Omega},
$$

where $A$ is the area circumscribed by the beams, $\lambda$ the wavelength and $P$ the perimeter of the gyro contour. The inner product accounts for the projection of the axis of rotation on the normal vector on the laser plane. Since ROMY has the shape of an inverted tetrahedron, each ring has a different projection angle to the Earth's rotation axis. Table 1 lists the respective Sagnac beat notes for all four rings with a sample spectrum for the horizontal ring shown in Fig. 5.

Table 1: The Sagnac beat frequencies (in Hz) obtained for all four components of the ROMY tetrahedron.

\begin{tabular}{cccc} 
Horizontal ring & West ring & North ring & East ring \\
\hline 553.5 & 440.4 & 305.3 & 439.9 \\
\hline
\end{tabular}

Each ring laser is operated at low beam powers of approximately $20 \mathrm{nW}$ in order to obtain a stable interferogram. After mixing the two counter-propagating laser beams in a beam combiner, the beat note is detected by a photo-multiplier through the application of a trans-impedance amplifier, then digitized. The resultant waveform of all four rings is digitized at a rate of $5 \mathrm{kHz}$ by a 24-bit digitizer unit (Kinemetrics Obsidian System). The analog-to-digital processing flow is described in the section 5.1. 


\subsection{Unequal Mode Indices and Drifts}

Each of the four ring cavities in ROMY has a perimeter over $30 \mathrm{~m}$, which means that adjacent longitudinal laser modes show a free spectral range of only $8.3 \mathrm{MHz}$ for the horizontal ring and $8.9 \mathrm{MHz}$ for the other rings. Although overpressuring of the resonator with helium suppresses the simultaneous excitation of several adjacent longitudinal laser modes and thus mode competition, it cannot avoid laser oscillation on different neighboring longitudinal mode indices for each sense of propagation. Although the cavity does not loose the ability to sense rotation, the interferogram is biased by the free spectral range into the regime of $8-16 \mathrm{MHz}$, which is outside the detection bandwidth of our data logging system. In practice, the rotation rate signal disappears from the data logger. ROMY is set up such, that a mode jump like this is detected by a watchdog system on the data logger. In order to recover the interferogram quickly, the recovery procedure raises the laser power above the multi-mode threshold and then drops the intensity level back to the preset values, thus providing the chance that the cavity settles down such, that both laser modes operate on the same longitudinal mode index. While this often recovers the gyroscope operations quickly, the process has to be repeated several times on occasion.

Figure 6 shows an example from the horizontal ring of ROMY. Due to the fact that the cavity length is not stabilized, the interferogram showing the Earth rotation rate is slightly drifting. The interferogram jumped to different oscillating laser modes several times during the shown measurement series, which also changed the magnitude of the systematic biases from backscatter coupling (Schreiber and Wells, 2013) and dispersion effects. The system recovered quickly most of the time, but three times throughout this day, the recovery process had to be applied several times before the measurement signal returned in the window of detection. Obviously this deteriorates the usability of the observations in particular for low-frequency signals. It is important to note that the potential for slight geometry changes was accepted in the design phase with the 
knowledge that strategies exist to stabilize these effects in a second construction phase (see Discussion).

\section{ROMY: Data Analysis for Geodesy and Geophysics}

In this section we present the principles of the frequency-demodulation technique leading to the rotation-rate time series used in geodesy and geophysics. We document the performance of the ring laser components using the concepts of Allan deviation and power-spectral densities. Finally we show observations of local, regional, and global seismic wavefields.

\subsection{Data Acquisition and Processing}

The Sagnac Eq. 1 suggests that the rotation-rate signal at the different rings are extracted best as a classical frequency demodulation. A schematic flow chart of the data acquisition and processing is provided in Fig. 7. In our case the carrier frequency or beat note is the (quasi-) constant Earth rotation rate projected on the area normal of the corresponding ring laser component. Additional contributions of (local) rotational ground motions like ocean generated ground motions, earthquake-induced signals, or anthropogenic noise will slightly alter this carrier frequency. The amount of this frequency modulation together with the timing of these changes translates directly into the amplitude-time trace of the rotation rate signal. It is important to note that the ground motion alters the rate of rotation, experienced by each interferometer in inertial space. We are not looking at a perturbation of the instrument. Eq. 1 tells us furthermore, that the Sagnac frequency scales with the size (area divided by circumference) and the orientation with respect to the Earth's rotation vector. As a consequence the carrier frequency (constant rotation rate of the Earth) increases with the size of the ring and as the cavity normal vector is increasingly aligned with the Earth's rotation vector, thus giving better resolution.

Given the tetrahedral ROMY setup we have to deal with carrier frequencies between 300-554 Hz (Table 1) 
with the highest frequency signal originating from the horizontal ring (vertical normal). In order to allow a precise and broad-band rotation rate signal reconstruction, these carrier frequencies have to be sampled with a sufficiently high data rate. A sampling frequency of $5 \mathrm{kHz}$ is chosen for all Sagnac channels. The high sampling rate - resulting in a large amount of raw data to be transmitted in real time - is essential for the successful demodulation of the rotation rate signal. In order to keep the time base of the sampling as consistent as possible, a 24 channel 24-bit digitizer of the Granite family (Obsidian, Kinemetrics) was chosen.

Next to the four Sagnac beat note channels $(\mathrm{Z}, \mathrm{U}, \mathrm{V}, \mathrm{W})$, there are provisions to sample the intensity of the eight laser beam channels (the individual counter-propagating beams) with the same digitizer and the same sampling rate. The remaining 12 channels are reserved for a co-located seismometer, tiltmeter, and environmental instruments (temperature, pressure, humidity). The data is transmitted in real time to a seedlink ring-server on which a plugin for near-real time conversion (demodulation) from Sagnac frequency to rotational motion is implemented. As seismologists are mainly interested in the frequency band between $0.001-10 \mathrm{~Hz}$ and the demodulation should be fast and real-time, the demodulation is done classically by estimating the real and quadrature phase of the Sagnac signal using a Hilbert transform.

Before the application of the Hilbert transform and the subsequent estimation of the instantaneous frequency (i.e. the rotation rate signal), the incoming data stream is collected to form batches of $1600 \mathrm{~s}$ of raw, continuous Sagnac-frequency data. This results in a longest usable period of nominally $800 \mathrm{~s}$ and will have to be modified when investigating free oscillations of the Earth. These data chunks are subsequently zero-phase bandpass filtered and up-sampled to $10 \mathrm{kHz}$. A Butterworth bandpass filter reduces possible side band noise effects. It also performs the required interpolation during the up-sampling process. In order to avoid artifacts of the filters, the impulse response at 20 times the time-frequency product of the bandpass filter is removed at the beginning and the end of the $1600 \mathrm{~s}$ signal segment. This up-sampled and filtered signal is then convolved with a truncated time-domain Hilbert filter (Dave Hale, Colorado School of Mines, 06/02/89). The instantaneous 
frequency is finally estimated by the approximation:

$$
f=\frac{x(t) d H[x(t)] / d t-d x(t) / d t H[x(t)]}{2 \pi\left(x(t)^{2}+H[x(t)]^{2}\right)},
$$

where $\mathrm{f}$ is the instantaneous frequency (i.e. the rotation rate), $\mathrm{x}(\mathrm{t})$ the Sagnac signal and $\mathrm{H}[]$ the Hilbert transform; d/dt stands for the time derivative.

In order to keep the resolution as high as possible but still use an efficient compression algorithm a constant offset value (i.e. constant part of the rotation rate of the Earth) is removed and the remaining numbers are scaled to form integers in multiples of $1 \mu \mathrm{Hz}$. These integers are further processed by seedlink plugins which apply a Steim 2 compression as well as subsequent down-sampling to seismologically usable sampling rates (i.e. 100, 20, $2 \mathrm{~Hz}$, respectively). Despite this very complex procedure the associated algorithm is fast enough to act in real-time and serves well for the most common seismological applications. In the case of very low and very high frequencies, however, the raw data has to be treated in different ways and in an off-line mode.

\subsection{Performance}

The performance characteristics of a ring-laser gyroscope are commonly described in terms of the Allan deviation (Allan, 1966). Originally designed for the performance characterization of high-precision oscillators, the Allan deviation $\sigma(\tau)$ can be calculated as follows:

$$
\sigma^{2}(\tau)=\left\langle\frac{\left(\bar{y}_{k+1}(\tau)-\bar{y}_{k}(\tau)\right)^{2}}{2}\right\rangle
$$

with $\bar{y}_{k}(\tau)$ as the $\mathrm{k}$-th average value of the time series $y$ of length $\tau$ and \langle\rangle denoting the average over all $\mathrm{k}$ along the time series $y$. The Allan deviation describes the resolution of the sensor readout after averaging over the time $\operatorname{span} \tau$.

According to Fig. 8, the ROMY Z-component and the V-component show a minimum in Allan deviation of $2.0 \mathrm{prad} / \mathrm{s}$ and $2.8 \mathrm{prad} / \mathrm{s}$, respectively, at an averaging time of $100 \mathrm{~s}$. The $\mathrm{W}$-component shows the minimum 
of $7 \mathrm{prad} / \mathrm{s}$ at $70 \mathrm{~s}$ averaging time while the U-component shows a minimum of approximately $8 \mathrm{prad} / \mathrm{s}$ at $400 \mathrm{~s}$ averaging time. As a consequence, the best performing ring (the Z-component) can resolve a rotation rate as low as $2 \mathrm{prad} / \mathrm{s}$ after averaging over $100 \mathrm{~s}$. For the best performing rings, the sensitivity at $1 \mathrm{~Hz}$ (averaging time of $1 \mathrm{~s}$ ) is between $80 \mathrm{prad} / \mathrm{s}$ and $100 \mathrm{prad} / \mathrm{s}$ which is still exceptional. At this point in time, the performance at lower frequencies $(<0.01 \mathrm{~Hz})$ is limited by the lack of a geometric stabilization system that can maintain the length of the cavity at all times. Due to the fact that the ROMY cavities are still unconstrained, the Allan deviation diverges after about $100 \mathrm{~s}$ as the optical frequency in the cavity drifts and non-reciprocal cavity effects cause a measurement bias.

For seismologists, a more common way to characterize the overall station performance in terms of background noise is the concept of probabilistic power spectral density (PPSD). Fig. 9 demonstrates the station performance at low levels of background noise. In order to exclude strong signals from nearby noise sources like farming machinery, we manually picked around 30 continuous recordings (for each ring) each lasting 6 hours with peak signal amplitudes not exceeding $100 \mathrm{nrad} / \mathrm{s}$. Consistent with the Allan deviation analysis (Fig. 8), the Z-component (channel HJZ in Fig. 9) shows the lowest noise levels over a period range from $1000 \mathrm{~s}$ to $10 \mathrm{~s}$. Note that the median of the PPSD distribution for the Z-component does not exceed a level of $20 \mathrm{prad} / \mathrm{s} / \sqrt{\mathrm{Hz}}$ in that period range. However, noise levels up to $1 \mathrm{nrad} / \mathrm{s} / \sqrt{\mathrm{Hz}}$ for periods of $1000 \mathrm{~s}$ most likely reflect temperature, pressure and construction settling effects acting on the unconstrained cavities. In the high frequency part of the background noise spectrum (above $1 \mathrm{~Hz}$ ), all four ROMY components behave similarly. Anthropogenic noise sources like the main road and the railway track passing nearby cause peak amplitude levels of $2 \mathrm{nrad} / \mathrm{s} / \sqrt{\mathrm{Hz}}$ at $10 \mathrm{~Hz}$. We want to point out here, that all four ROMY components clearly see ocean generated microseismic noise at frequencies between $0.2 \mathrm{~Hz}$ and $0.3 \mathrm{~Hz}$. 


\subsection{Earth's Rotation}

ROMY has twice the scale factor (the proportional factor in Eq. 1) of the G ring laser. So it is sensitive enough to measure variations in the rate of Earth rotation, provided that the stability of the entire installation can be improved to take the knee of the Allan deviation of Fig. 8 down to 1 part in $10^{9}$ of Earth's rotation. Expressed differently, a laser gyro for Earth rotation monitoring has to resolve a rotation rate of less than $0.01 \mathrm{prad} / \mathrm{s}$. Furthermore, it has to remain stable for several weeks. ROMY as opposed to any of the other single component large ring lasers existing today, can resolve the complete Earth rotation vector in a selfcontained fashion, because all three components of rotation in space are captured by the individual rings. On top of that, there is one extra ring available, which offers redundancy to check for consistent operation.

At appropriate resolution the ground-based observations of the Earth's full rotation vector certainly would be a desired complement to VLBI (e.g., Mendes Cerveira et al., 2009). Gebauer et al. (2020) report a first discussion of the initial ROMY ring laser performance with respect to geodetic requirements. Over a length of 47 days, a long-term sensor stability of $\Delta \Omega / \Omega=5 \times 10^{-5}$ - where $\Omega$ is Earth's rotation rate - could be achieved. In an Earth-centered frame of reference this corresponds to an orientation change of the rotation axis of around $0.1 \mathrm{asec}$, which translates into $\approx 3 \mathrm{~m}$ of polar motion. While this is the most accurate direct measurement of the Earth's complete rotation vector by a ring laser, it clearly needs improvement. This can be achieved by the full implementation of the cavity stabilization procedure and is planned for the near future.

\subsection{Earthquake-induced Ground Motions}

The recording and analysis of broadband rotational ground motions is a recent, but emerging field and most previous observational studies were limited to either single-component ring laser systems (e.g., Igel et al., 2005; Cochard et al., 2006; Igel et al., 2007), or array-derived rotational motions (e.g., Huang, 2003). An 
event data base exists (Salvermoser et al., 2017) into which seismic events recorded on the G-ring laser and - as of recently - the ROMY ring laser are written on a daily basis. In the past few years portable broadband sensors that measure rotational ground motions have been developed (Bernauer et al., 2018) and are now being applied in the field (e.g., Yuan et al., 2020b; Wassermann et al., 2020). However, it is important to note that they are approximately three orders of magnitude less sensitive than the ring laser systems, therefore less capable of capturing regular global earthquake-induced wavefields.

In the following sections we will show exemplary seismic observations from the classic seismic distance categories. A detailed analysis will be provided in a follow-up study. The innovation for seismology with the ROMY ring laser (compared to previous ring laser observations) is the possibility to observe directly the horizontal components of rotational motions (i.e., tilt rate) allowing also the analysis of P-SV (and Rayleigh surface wave) motions with unprecedented accuracy.

\subsubsection{Teleseismic Event}

In Fig. 10 ground motion observations recorded in Fürstenfeldbruck, Germany, with the seismic broadband (STS2) station FUR and the ROMY ring laser (at a distance of approximately $20 \mathrm{~m}$ ) are shown following the M7.6 earthquake in the region of Papua New Guinea on May 14th 2019. The epicentral distance was $125^{\circ}$ $(\approx 14000 \mathrm{~km})$. The seismometer data were rotated into a local $R T V$ (radial-transverse-vertical) system, and the original velocity data were instrument-corrected and converted to ground acceleration. The 4-component ROMY ringlaser data were combined to a $Z N E$ system and then also rotated into a $R T Z$ system. All traces were bandpass-filtered in the interval $[0.01-0.1 \mathrm{~Hz}]$.

In Fig. 10b-d the vertical component of the ground acceleration $A z$ and the transverse component of the ground rotation rate $R t$ (multiplied by a factor -1 to achieve phase match) are analysed. The traces of acceleration (black) and rotation rate (red) are superimposed, the arrival time of the $S S$ phase is marked. Across 
almost the entire time window the phase match between both traces is (visually) excellent indicating that the wave fronts are close to planar and that body wave P-SV motions and Rayleigh wave motions are correlated as expected from simple plane wave theory (e.g., Li et al., 2002). The amplitude match between rotation rate and vertical acceleration is achieved by scaling with an apparent horizontal phase velocity of $\approx 3 \mathrm{~km} / \mathrm{s}$.

To further characterize the teleseismic ground motions we calculate the correlation coefficient between vertical acceleration and rotation rate as a function of assumed backazimuth in a $50 \mathrm{~s}$ sliding time window. The maximum correlation is denoted by a black dot that scatters around the theoretical backazimuth (dashed blue line) and is very stable in the expected propagation direction in the window containing the Rayleigh waves. Apparent phase velocities are estimated whenever the wave-form match exceeds 0.95 correlation coefficient (Fig. 10d). The high phase velocities around the $S S$ arrival are due to the steep incidence angles of P-SV body-wave phases. The highest correlations (color-coded) are observed in the time window containing the Rayleigh wave energy with phase velocities between 2.5 and $4.0 \mathrm{~km} / \mathrm{s}$.

In Fig. 10e-g the corresponding analysis is carried out for the vertical component of rotational motions $R z$ and the transverse acceleration $A t$, as was previously done using data from the G-ring laser (e.g., Igel et al., 2007). In this case the ring laser is sensitive to $S H$-type motions only. Prior to the $S S$ arrival there is substantially less energy in the vertical rotation than in the horizontal component (discussed above), as expected for a predominantly spherically symmetric Earth. The backazimuth estimation is stable almost along the entire seismograms. The indication of surface wave dispersion in the window containing the Love waves is more apparent than for the Rayleigh wave case. However, it is important to note that the phase velocity estimate can be affected by the superposition of higher surface wave modes (e.g., Kurrle et al., 2010). 


\subsubsection{Regional Seismic Event}

An example of a regional seismic event at a distance of around $1500 \mathrm{~km}$ that occurred in Turkey September 26, 2019 with a magnitude $\mathbf{M}_{w} 5.7$ is shown in Fig. 11. The processing and graphical representation is identical to the teleseismic event. The data have been bandpassed in the interval $[0.01-0.2 H z]$. The waveform fit between appropriately rotated acceleration and rotation rate signals is less pronounced than in the teleseismic case. Due to the higher frequencies involved we expect stronger effects due to non-planar wavefronts and scattering in general. There is also consequently more scattering of the back-azimuth that has highest correlation and there seems to be a systematic shift away from the true backazimuth in both SH and P-SV type setups (except for some time windows with very high correlations).

While the Rayleigh wave phase velocity estimates in the time windows with high correlations (e.g., $t=610 \mathrm{~s}$ ) are comparable with those for the teleseismic event, the Love wave phase velocity estimates (Fig. 11g), are estimated at the lower end of the correlation scale and are questionable.

\subsubsection{Local Seismic Event}

A comparably strong local event occurred at a distance of $144 \mathrm{~km}$ south of the Germany-Austria border with a local magnitude of $M_{L} 3.8$ on February 1, 2018, with results shown in Fig. 12. The data were rotated accordingly and bandpass-filtered in the interval $[0.01-1 H z]$. Note that the dominant frequency here is substantially higher than for the event in Turkey. Nevertheless, the waveform correlation in both P-SV and SH cases is high, and the point of maximum correlation as a function of backazimuth captures fairly well the actual backazimuth direction.

In this local event case the correlation of the SH-type motion seems consistently higher compared to the $\mathrm{P}-\mathrm{SV}$ case. What is remarkable is the lack of energy in the SH case prior to the S-wave arrival. Whether the low Love-wave phase velocities of around $1.5 \mathrm{~km} / \mathrm{s}$ are compatible with local velocity estimates remains to be 
checked. The phase velocity estimates in the P-SV case are - similar to the case discussed above - at the lower end of the correlation window and should be treated with care.

\subsection{Ocean-generated Noise}

One of the outstanding questions on ocean-generated seismic noise (microseisms) is how much Rayleigh waves and Love waves are contained in the primary microseism (approximately in the range $0.05-0.07 \mathrm{~Hz}$ ) and in the secondary microseism (approximately $0.10-0.40 \mathrm{~Hz}$ ). A precise answer to this question is surprisingly difficult because the amount of Love waves is hard to estimate. While vertical component seismograms provide us clean records for Rayleigh waves, horizontal component seismograms contain both Rayleigh and Love waves and their separation is not necessarily easy unless an array of seismometers is available.

The vertical rotation data from a ring laser can provide a unique dataset to address this question because vertical rotation data predominantly consist of Love waves. We have combined the ring laser data with station naming RLAS with vertical seismic data at Wettzell to estimate the amount of Love-wave energy in the secondary microseism that is passing through the station (Tanimoto et al., 2015, 2016). The amount of Love waves was surprisingly large at Wettzell, comparable or slightly larger than the amount of Rayleigh waves throughout a year.

New ROMY data, recorded about $200 \mathrm{~km}$ to the southwest of Wettzell, provide another opportunity to validate this estimate. Comparison of vertical rotation data from RLAS (Wettzell) and ROMY are shown in Fig. 13, plotted for the entire year of 2018. The top panel is a time-frequency plot for the vertical rotation from ROMY, plotted from 0 to $1 \mathrm{~Hz}$ in the vertical axis. The horizontal axis is the Julian Day. The bottom panel is a similar time-frequency plot for RLAS. Power spectral densities of rotation are plotted in color; high amplitudes (brown and red) are seen for frequencies between about $0.10 \mathrm{~Hz}$ and $0.40 \mathrm{~Hz}$ in both data sets. They show seasonal variations, typically small amplitudes in summer and large amplitudes in winter. These 
are signals of the ocean-generated secondary microseism.

There is a distinct difference in amplitudes between the two stations, however. Color scales on the righthand side of plots (Fig. 13) indicate that PSDs from ROMY are about 50-100 times larger than those at RLAS (Wettzell). This must be related to elastic properties at shallow depths. The site of ROMY is located in an alluvial basin (glacial deposits) while Wettzell - the site of a VLBI station - is characterized by igneous rocks.

ROMY can also provide horizontal rotation data (i.e., tilt). They are the direct measurements of tilt which would be primarily composed of Rayleigh-wave signals. With three component rotation data at ROMY, in contrast to only vertical rotation data from RLAS, we will be able to analyze new aspects of ocean-generated seismic noise from ROMY data and deepen our understanding of this natural source.

\section{Discussion and Conclusion}

ROMY is an acronym for ROtational Motions in seismolog $Y$, the name of the EU-funded ERC-Adv project that funded the construction of the first multi-component ring laser. It is also an indication that originally the ring laser was supposed to primarily serve as the most sensitive ground rotation recording instrument world wide. Through further funding, the original dimensions of the tetrahedral-shaped ring laser system $(6 \mathrm{~m}$ side length) could be extended to $12 \mathrm{~m}$ thereby making it a) a very interesting sensor for geodesy as the theoretical sensitivity exceeds the currently most accurate system, the G-ring; b) more prone to instabilities due to the difficulties in stabilizing the larger geometry; and c) a grand challenge as far as the construction was concerned and the establishment of the optical paths in the triangular cavities.

In the light of these challenges, the successful construction of the first multi-component ring laser of this shape and kind can be considered a substantial progress in ring laser technology applicable to geodesy and geophysics. The level of accuracy of the concrete construction was such that the mechanical degrees of freedom of the ring laser hardware (in particular the corner boxes and the movable mirrors) where such that for 
all four rings the optical paths could be established allowing the Sagnac effect to be observed. As a consequence the ground-based reconstruction of the entire vector of Earth rotation was possible with high accuracy (Gebauer et al., 2020). For seismology, ROMY - combined with the collocated broadband STS2 sensor (FUR) - constitutes the most accurate 6 degree-of-freedom point measurement to date. The ultimate goal of ground motion (including the rotational component) measurements is to observe below the physical low-noise model of planet Earth. While this goal has been achieved with translational sensors decades ago, for the rotation component this still constitutes a formidable problem. ROMY - with ocean generated noise clearly observed is a major step in this direction. Portable sensors (e.g., Bernauer et al., 2018) will likely not be able to achieve this goal in the foreseeable future.

An important aspect of the specific ROMY design was that tiny geometry changes due to thermal or other effects were taken into account. These usually long-term effects primarily affect geodetic or very long-period seismic observations. An actual monolithic structure (as implemented for the G-ring) would have been prohibitively expensive. However, with further modifications to the current system (e.g., locking the optical frequency of each ring laser to an optical reference and adjusting the laser cavity through mirror movements, e.g., Schreiber and Wells (2013)) a virtual monolithic structure shall be established.

As indicated in the technical sections above, ROMY is a delicate instrument with a number of problems that affect the quality of the observations. These include effects of deterioration of the gas mixture, a stable optical frequency and equal laser beam power in the cavities established via a feedback loops, and effects from slight settling of the monument construction affecting the rigid structure and thus the optical path. These problems make it currently difficult to obtain continuous undisturbed multi-component observations that can be combined to generate the desired 3C local ground rotations and Earth's rotation vector. However, none of these problems are insurmountable.

With these improvements implemented, we expect stable long-term observations, which will allow recov- 
ery of variations in the length-of-day, a high resolution time series of the Earth rotation vector in order to fuse them with VLBI measurements. In addition we expect the routine observation of local to global seismicity, ocean generated noise, and Earth's free oscillations excited by large earthquakes or infragravity waves. Furthermore, the ROMY system can be used for the investigation of 6 degree-of-freedom point seismic processing schemes, such as local seismic velocity analysis (e.g., Wassermann et al., 2016; Keil et al., 2020), the comparison with array-derived rotation that is installed around ROMY (e.g., Suryanto et al., 2006; Donner et al., 2017), or the tracking of seismic sources (e.g., Yuan et al., 2020a).

\section{Acknowledgements}

The ROMY ring laser has been funded by an ERC-Advanced Grant of the European Research Council (ROMYproject, grant number 339991). We are grateful to the leadership of the Ludwig-Maximilians University providing substantial additional support for the construction of ROMY. We thank the Staedtische Bauamt II for their support. We thank the Bundesamt für Kartographie and Geodäsie for their support. We thank Stefanie Schwartz for her help with the ring laser hardware, Jonas Igel for the photo sessions, Monika Terzjak for her theoretical orientation analysis. We thank the technical staff of the partner at the University of Canterbury, New-Zealand, for their help.

\section{References}

Aki, K. and Richards, P. (2002). Quantitative Seismology. University Science Books.

Allan, D. W. (1966). Statistics of atomic frequency standards. Proceedings of the IEEE, 54(2):221-230.

Bernauer, F., Wassermann, J., Guattari, F., Frenois, A., Bigueur, A., Gaillot, A., de Toldi, E., Ponceau, D., 
Schreiber, U., and Igel, H. (2018). BlueSeis3A: Full Characterization of a 3C Broadband Rotational Seismometer. Seismological Research Letters, 89(2A):620-629.

Bernauer, F., Wassermann, J., and Igel, H. (2012). Rotational sensors-a comparison of different sensor types. Journal of Seismology, 16(4):595-602.

Bernauer, F., Wassermann, J., and Igel, H. (2020). Dynamic Tilt Correction Using Direct Rotational Motion Measurements. Seismological Research Letters.

Brokesova, J., Malek, J., and Kolinsky, P. (2012). Rotaphone, a mechanical seismic sensor system for field rotation rate measurements and its in situ calibration. Journal of Seismology, 16(4, SI):603-621.

Cochard, A., Igel, H., Schuberth, B., Suryanto, W., Velikoseltsev, A., Schreiber, U., Wassermann, J., Scherbaum, F., and Vollmer, D. (2006). Rotational motions in seismology: Theory, observation, simulation. In Teisseyre, R., Majewski, E., and Takeo, M., editors, Earthquake Source Asymmetry, Structural Media and Rotation Effects, pages 391-411. Springer Berlin Heidelberg.

Donner, S., Bernauer, M., and Igel, H. (2016). Inversion for seismic moment tensors combining translational and rotational ground motions. Geophysical Journal International, 207(1):562-570.

Donner, S., Lin, C., Hadziioannou, C., Gebauer, A., Vernon, F., Agnew, D. C., Igel, H., Schreiber, U., and Wassermann, J. (2017). Comparing Direct Observation of Strain, Rotation, and Displacement with Array Estimates at Piñon Flat Observatory, California. Seismological Research Letters, 88(4):1107-1116.

Dunn, R. W., Shabalin, D. E., Thirkettle, R. J., MacDonald, G. J., Stedman, G., and Schreiber, K. U. (2002). Design and initial operation of a 367-m2 rectangular ring laser. Applied Optics, 41:1685-1688.

Edme, P. and Yuan, S. (2016). Local dispersion curve estimation from seismic ambient noise using spatial gradients. Interpretation, 4(3):SJ17-SJ27. 
Gebauer, A., Schreiber, K., Kluegel, T., Schoen, N., and Ulbrich, U. (2012). High-frequency noise caused by wind in large ring laser gyroscope data. Journal of Seismology, 16(4):777-786.

Gebauer, A., Tercjak, M., Schreiber, K. U., Kodet, J., Hugentobler, U., Igel, H., Wassermann, J., Bernauer, F., Lin, C. J., Egdorf, S., Simonelli, A., and Wells, J.-P. R. (2020). All optical reconstruction of the instantaneous earth rotation vector from a large scale laser gyroscopic array. Physical Review Letters, XX.

Hand, E. (2017). Lord of the rings. Science, 356(6335):236-238.

Huang, B.-S. (2003). Ground rotational motions of the 1999 Chi-Chi, Taiwan earthquake as inferred from dense array observations. Geophysical Research Letters, 30(6).

Hurst, R. B., Dunn, R. W., Schreiber, K. U., Thirkettle, R. J., and MacDonald, G. K. (2004). Mode behavior in ultralarge ring lasers. Appl. Opt, 43(11):2337-2346.

Igel, H., Bernauer, M., Wassermann, J., and Schreiber, K. U. (2015). Rotational Seismology: Theory, Instrumentation, Observations, Applications. Encyclopedia of Complexity and Systems Science, Springer-Verlag New York.

Igel, H., Brokesova, J., Evans, J., and Zembaty, Z. (2012). Preface. Journal of Seismology, 16(4):571-572.

Igel, H., Cochard, A., Wassermann, J., Flaws, A., Schreiber, U., Velikoseltsev, A., and Pham Dinh, N. (2007). Broad-band observations of earthquake-induced rotational ground motions. Geophysical Journal International, 168(1):182-196.

Igel, H., Nader, M.-F., Kurrle, D., Ferreira, A. M. G., Wassermann, J., and Schreiber, K. U. (2011). Observations of Earth's toroidal free oscillations with a rotation sensor: The 2011 magnitude 9.0 Tohoku-Oki earthquake. Geophysical Research Letters, 38(21). 
Igel, H., Schreiber, U., Flaws, A., Schuberth, B., Velikoseltsev, A., and Cochard, A. (2005). Rotational motions induced by the M8.1 Tokachi-oki earthquake, September 25, 2003. Geophysical Research Letters, 32(8).

Jaroszewicz, L. R., Krajewski, Z., and Teisseyre, K. P. (2012). Usefulness of AFORS-autonomous fibre-optic rotational seismograph for investigation of rotational phenomena. Journal of Seismology, 16(4, SI):573-586.

Keil, S., Wassermann, J., and Igel, H. (2020). Single-station seismic microzonation using 6c measurements. Journal of Seismology.

Kurrle, D., Igel, H., Ferreira, A. M. G., Wassermann, J., and Schreiber, U. (2010). Can we estimate local love wave dispersion properties from collocated amplitude measurements of translations and rotations? Geophysical Research Letters, 37(4).

Lee, W. H. K., Celebi, M., Todorovska, M. I., and Igel, H. (2009). Introduction to the special issue on rotational seismology and engineering applications. Bulletin of the Seismological Society of America, 99(2B):945-957.

Li, H.-N., Sun, L.-Y., and Wang, S.-Y. (2002). Frequency dispersion characteristics of phase velocities in surface wave for rotational components of seismic motion. Journal of Sound and Vibration, 258(5):815 827.

Li, Z. and van der Baan, M. (2017). Tutorial on rotational seismology and its applications in exploration geophysics. GEOPHYSICS, 82(5):W17-W30.

Lindner, F., Wassermann, J., Schmidt-Aursch, M., Schreiber, K. U., and Igel, H. (2017). Seafloor ground rotation observations: potential for improving signal-to-noise ratio on horizontal obs components. Seismological Research Letters, 88(4):1-19.

Mendes Cerveira, P. J., Böhm, J., Schuh, H., Kluegel, T., Velikoseltsev, A., Schreiber, U., and Brzezinski, A. 
(2009). Earth rotation observed by very long baseline interferometry and ring laser. Pure appl. geophys, 166:1499-1517.

Nader, M., Igel, H., Ferreira, A., Kurrle, D., Wassermann, J., and Schreiber, K. (2012). Toroidal free oscillations of the earth observed by a ring laser system: a comparative study. Journal of Seismology, 16(4):745755.

Pritsch, B., Schreiber, K. U., Velikoseltsev, A., and Wells, J.-P. R. (2007). Scale-factor corrections in large ring lasers. Applied Physics Letters, 91(6):061115-061115-3.

Salvermoser, J., Hadziioannou, C., Hable, S., Krischer, L., Chow, B., Wassermann, J., Schreiber, U., Gebauer, A., and Igel, H. (2017). An Event Database for Rotational Seismology. Seismological Research Letters, $88(3)$.

Schmelzbach, C., Donner, S., Igel, H., Sollberger, D., Taufiqurrahman, T., Bernauer, F., Haeusler, M., Renterghem, C. V., Wassermann, J., and Robertsson, J. (2018). Advances in 6-c seismology: applications of combined translational 1 and rotational motion measurements in global and exploration seismology. Geophysics, 83(3):WC53-WC69.

Schreiber, K., Kluegel, T., Velikoseltsev, A., Schlueter, W., Stedman, G., and Wells, J.-P. (2009a). The Large Ring Laser G for Continuous Earth Rotation Monitoring. Pure and Applied Geophysics, 166(8-9):14851498.

Schreiber, K., Stedman, G., Igel, H., and Flaws, A. (2006a). Ring laser gyroscopes as rotation sensors for seismic wave studies. In Teisseyre, R., Majewski, E., and Takeo, M., editors, Earthquake Source Asymmetry, Structural Media and Rotation Effects, pages 377-390. Springer Berlin Heidelberg. 
Schreiber, K. U., Gebauer, A., Igel, H., Wassermann, J., Hurst, R. B., and Wells, J. P. R. (2014). The centennial of the sagnac experiment in the optical regime. Comptes Rendus Physique, 15(1):859-865.

Schreiber, K. U., Hautmann, J. N., Velikoseltsev, A., Wassermann, J., Igel, H., Otero, J., Vernon, F., and Wells, J.-P. R. (2009b). Ring laser measurements of ground rotations for seismology. Bulletin of the Seismological Society of America, 99(2B):1190-1198.

Schreiber, K. U., Klügel, T., Velikoseltsev, A., Schlüter, W., Stedman, G. E., and Wells, J.-P. R. (2009c). The large ring laser $\mathrm{g}$ for continuous earth rotation monitoring. Pure and Applied Geophysics, 166(8-9):14851498.

Schreiber, K. U., Klügel, T., Wells, J.-P. R., Hurst, R. B., and Gebauer, A. (2011). How to detect the chandler and the annual wobble of the earth with a large ring laser gyroscope. Physical Review Letters, 107(17).

Schreiber, K. U., Velikoseltsev, A., Carr, A. J., and Franco-Anaya, R. (2009d). The Application of Fiber Optic Gyroscopes for the Measurement of Rotations in Structural Engineering. Bulletin of the Seismological Society of America, 99(2B):1207-1214.

Schreiber, K. U., Velikoseltsev, A., Rothacher, M., Kluegel, T., Stedman, G. E., and Wiltshire, D. L. (2004). Direct measurement of diurnal polar motion by ring laser gyroscopes. Journal of Geophysical Research: Solid Earth, 109(B6).

Schreiber, K. U. and Wells, J.-P. R. (2013). Invited Review Article: Large ring lasers for rotation sensing. Review of Scientific Instruments, 84(4):041101-041101-26.

Schreiber, U., Igel, H., Cochard, A., Velikoseltsev, A., Flaws, A., Schuberth, B., Drewitz, W., and Mueller, F. (2006b). The GEOsensor Project: Rotations — a New Observable for Seismology. In Flury, J., Rummel, 
R., Reigber, C., Rothacher, M., Boedecker, G., and Schreiber, U., editors, Observation of the Earth System from Space, pages 427-443. Springer Berlin Heidelberg.

Schreiber, U., Klügel, T., and Stedman, G. E. (2003). Earth tide and tilt detection by a ring laser gyroscope. Journal of Geophysical Research, 108(B2).

Sollberger, D., Greenhalgh, S. A., Schmelzbach, C., Van Renterghem, C., and Robertsson, J. O. (2018). 6-c polarization analysis using point measurements of translational and rotational ground-motion: theory and applications. Geophysical Journal International, 213(1):77-97.

Suryanto, W., Igel, H., Wassermann, J., Cochard, A., Schuberth, B., Vollmer, D., Scherbaum, F., Schreiber, U., and Velikoseltsev, A. (2006). First comparison of array-derived rotational ground motions with direct ring laser measurements. Bulletin of the Seismological Society of America, 96(6):2059-2071.

Tanimoto, T., Hadziioannou, C., Igel, H., Wassermann, J., Schreiber, U., and Gebauer, A. (2015). Estimate of rayleigh-to-love wave ratio in the secondary microseism by colocated ring laser and seismograph. Geophys. Res. Lett., 42.

Tanimoto, T., Hadziioannou, C., Igel, H., Wassermann, J., Schreiber, U., Gebauer, A., and Chow, B. (2016). Seasonal variations in the rayleigh-to-love wave ratio in the secondary microseism from colocated ring laser and seismograph. J. Geophys. Res. Solid Earth, 121:2447-2459.

Trifunac, M. D. (2009). Review: Rotations in Structural Response. Bulletin of the Seismological Society of America, 99(2B):968-979.

Wassermann, J., Bernauer, F., Shiro, B., Johanson, I., Guattari, F., and Igel, H. (2020). Six-axis ground motion measurements of caldera collapse at kīlauea volcano, hawai' i-more data, more puzzles? Geophysical Research Letters, 47(5):e2019GL085999. 
Wassermann, J., Wietek, A., Hadziioannou, C., and Igel, H. (2016). Toward a single-station approach for microzonation: Using vertical rotation rate to estimate love-wave dispersion curves and direction finding. Bulletin of the Seismological Society of America, 106(3):1316-1330.

Widmer-Schnidrig, R. and Zuern, W. (2009). Perspectives for ring laser gyroscopes in low-frequency seismology. Bulletin of the Seismological Society of America, 99(2B):1199-1206.

Yuan, S., Gessele, K., Gabriel, A.-A., May, D. A., Wassermann, J., and Igel, H. (2020a). Seismic source tracking with six degree-of-freedom ground motion observations. Journal of Geophysical Research: Solid Earth, page submitted.

Yuan, S., Simonelli, A., Lin, C., Bernauer, F., Donner, S., Braun, T., Wassermann, J., and Igel, H. (2020b). Six Degree-of-Freedom Broadband Ground-Motion Observations with Portable Sensors: Validation, Local Earthquakes, and Signal Processing. Bulletin of the Seismological Society of America, 110(3):953-969. 
Figures 


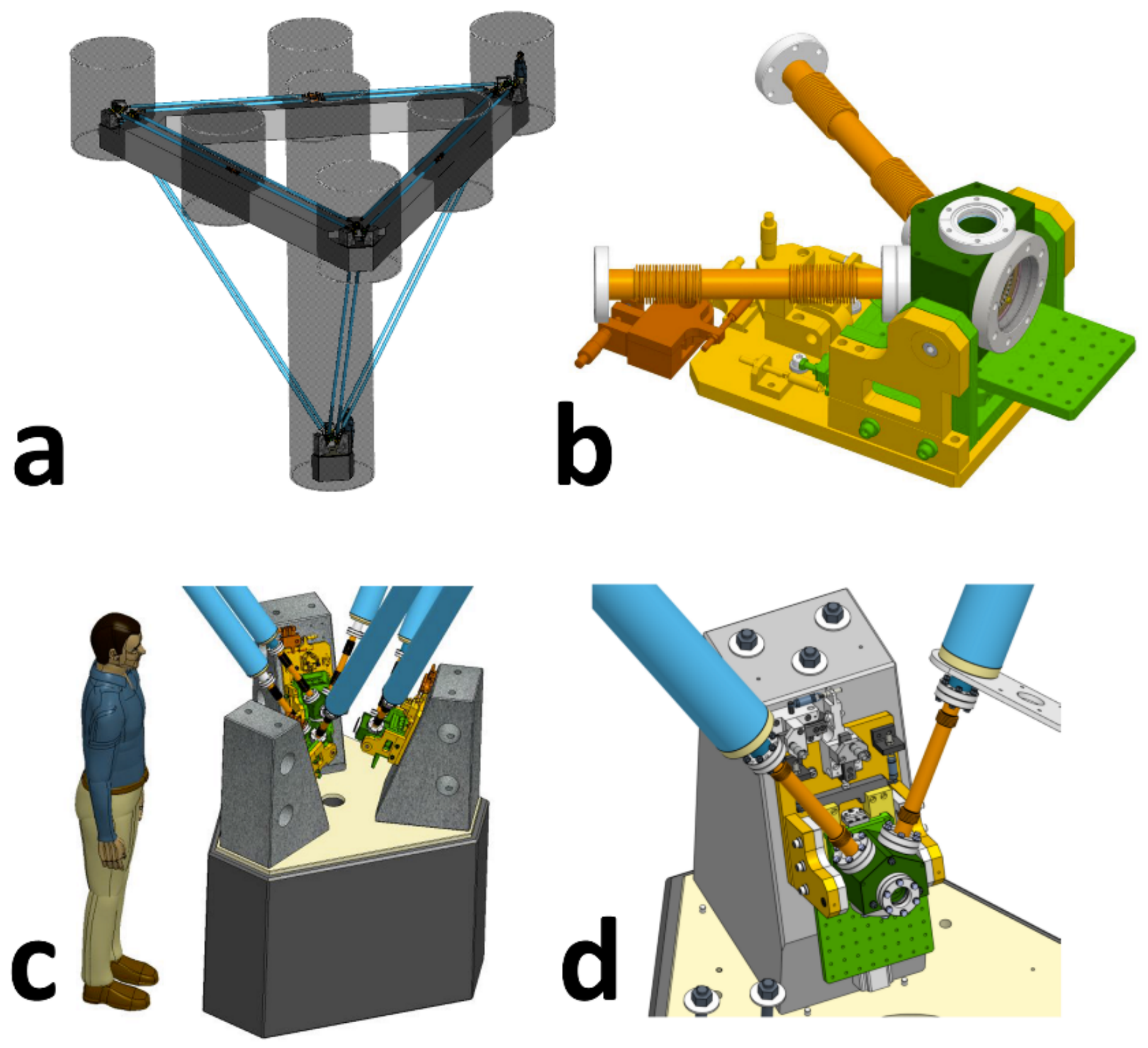

Figure 1: The ROMY geometry and hardware. a: Tetrahedral geometry of the ROMY ringlaser. The grey shaded cylinders illustrate the shafts that give access to ring laser corners and laser activation units. b: Drawing showing the vacuum tubes that contain the laser light (dark yellow) as well as the corner box with the reflective mirrors (green). The light intensity with the Sagnac signal can be measured through the rear window. c: The bottom of the ROMY structure with the corners of the three sub-horizontal ring lasers rigidly connected on a steel base plate attached to a concrete slab. d: Corner box of one of the sub-horizontal ring lasers. 

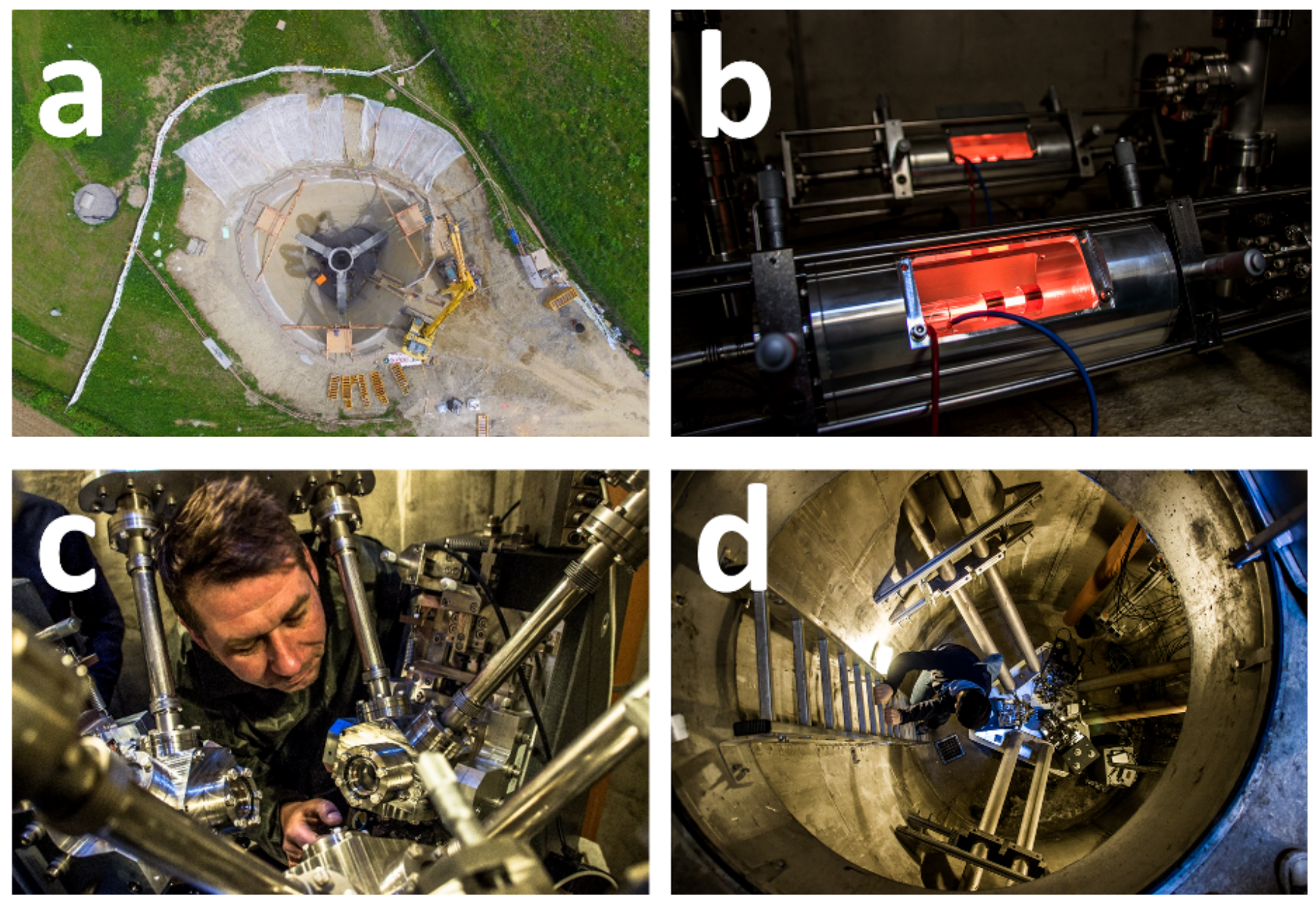

Figure 2: ROMY construction and final installation: a: Drone view of the excavated volume, the concrete hull, and the top-up construction of the tetrahedral structure (Photo: Fa. Wadle) b: Examples of laser light generation at the center of the horizontal side tubes. c: Setup of the rigid bottom plate with the corners of the three sub-horizontal ring lasers. d: View down the central shaft to the tip of the tetrahedron shown in c. (b-d photos: J. Igel) 


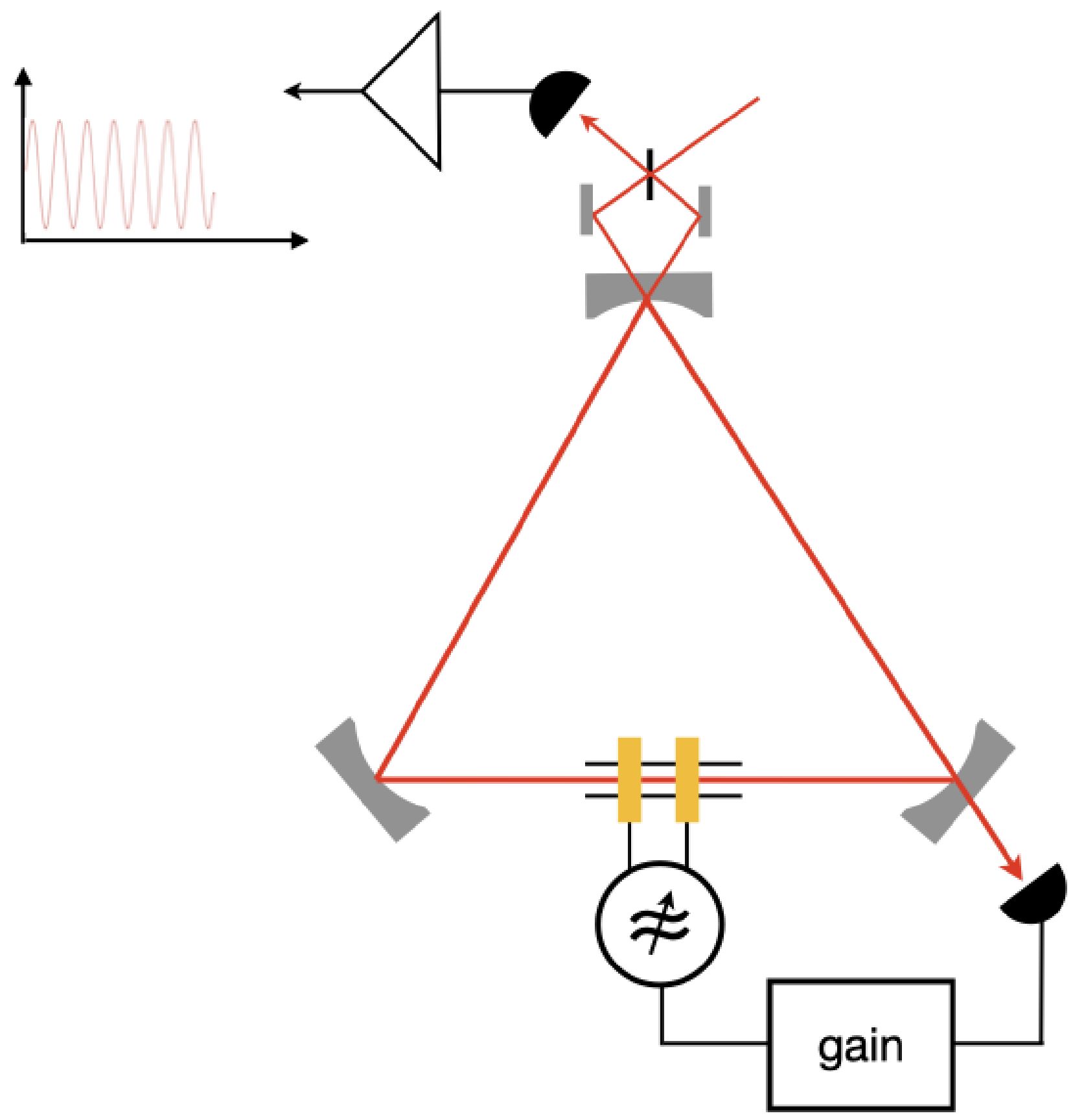

Figure 3: Schematic of the ring laser setup. Three curved mirrors form the cavity. A capillary on one side acts as a mode selector and provides the laser gain. Lasing is excited by RF-excitation of a plasma in the capillary. 


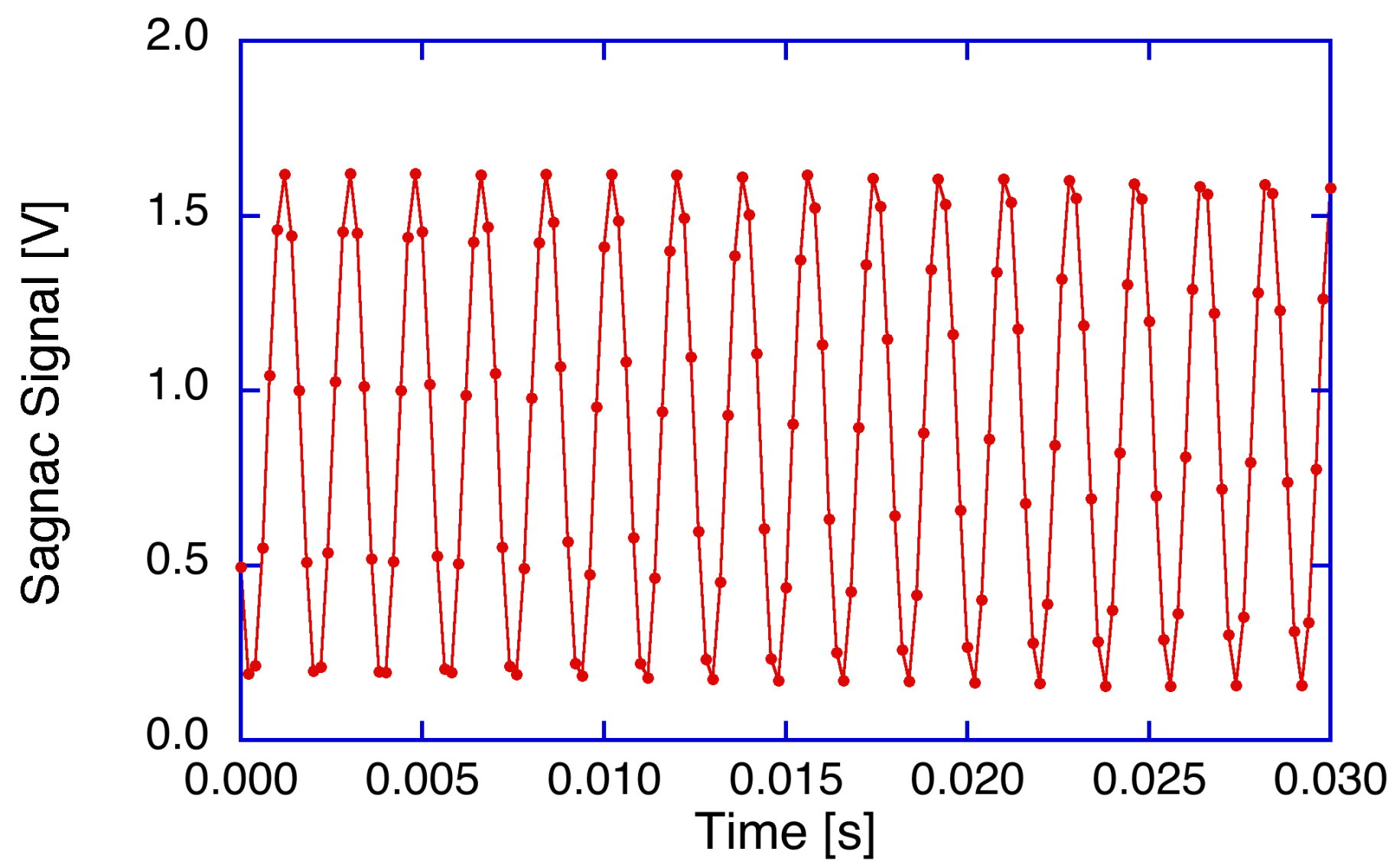

Figure 4: Example of the time domain signal obtained from the horizontal ring. The Sagnac beat note of $553.55 \mathrm{~Hz}$ is sampled by 24 bit digitizer at $5 \mathrm{kHz}$ sampling rate. 


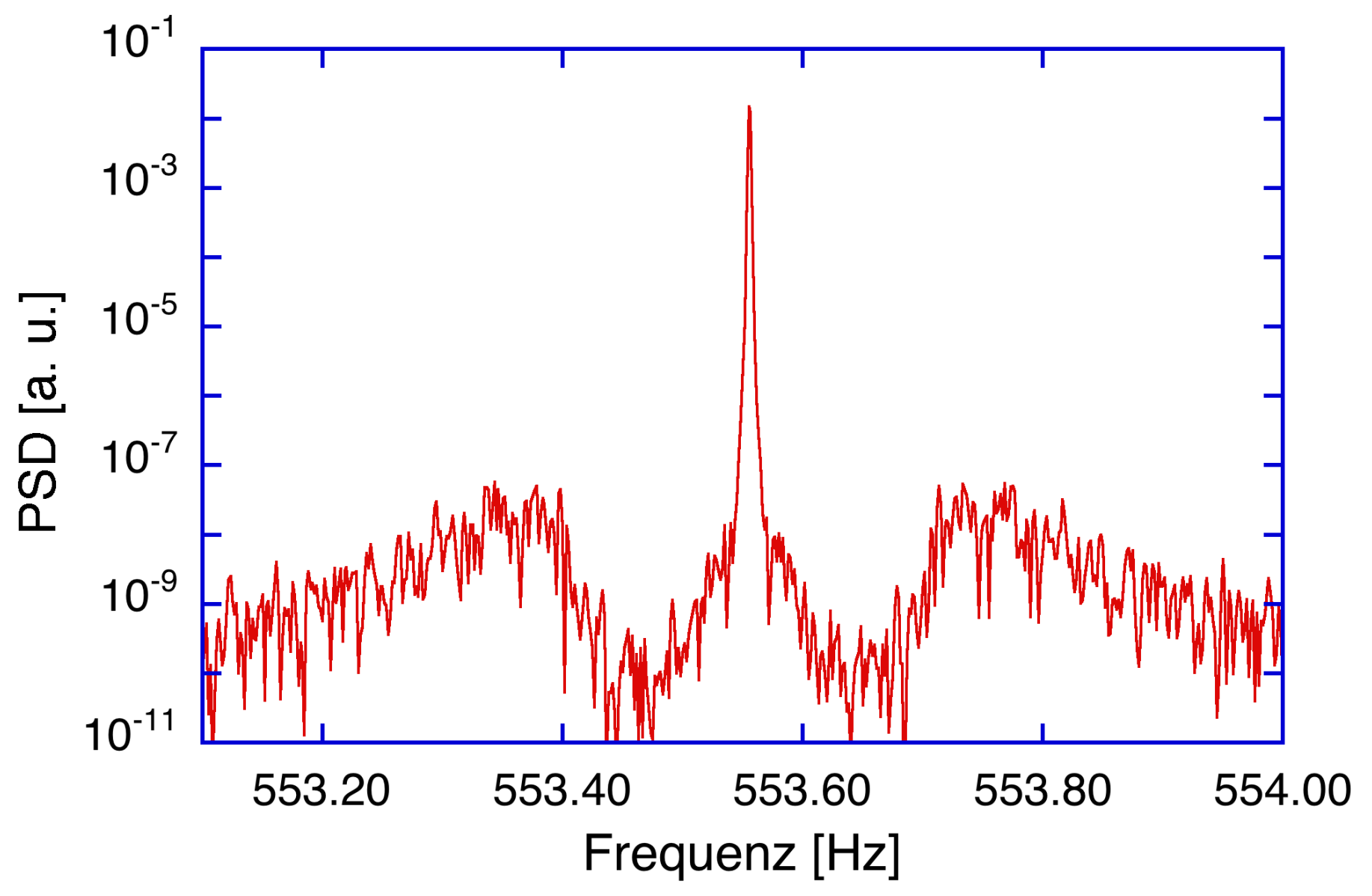

Figure 5: A sample spectrum of the ring laser beat note. The constant bias of the Earth rotation rate causes the beat note at 553.5 Hz. Geophysical signals from microseismic activity appear as a frequency modulation of this carrier at around $0.2 \mathrm{~Hz}$ on either side of the main peak. 


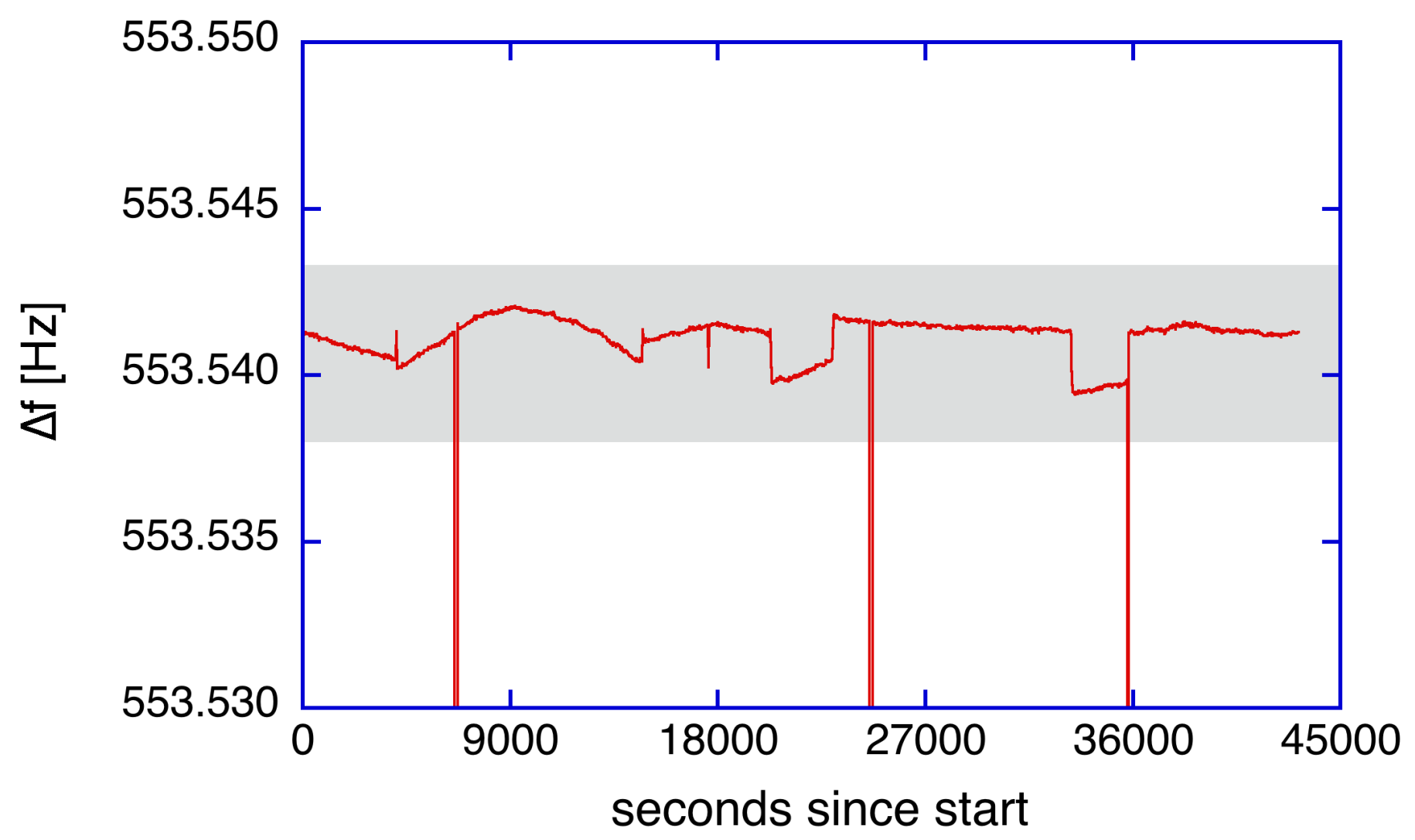

Figure 6: Example of a typical time series of the Earth rate bias. Since the laser resonator is not length stabilized, the measured Earth rate exhibits a small drift. The steps in the signal indicate when a mode-jump recovery occurred. At three times during this measurement the recovery process lasted notably longer. The gray-shaded area indicates the range of expected frequency variations due to changes in Earth's rotation. 


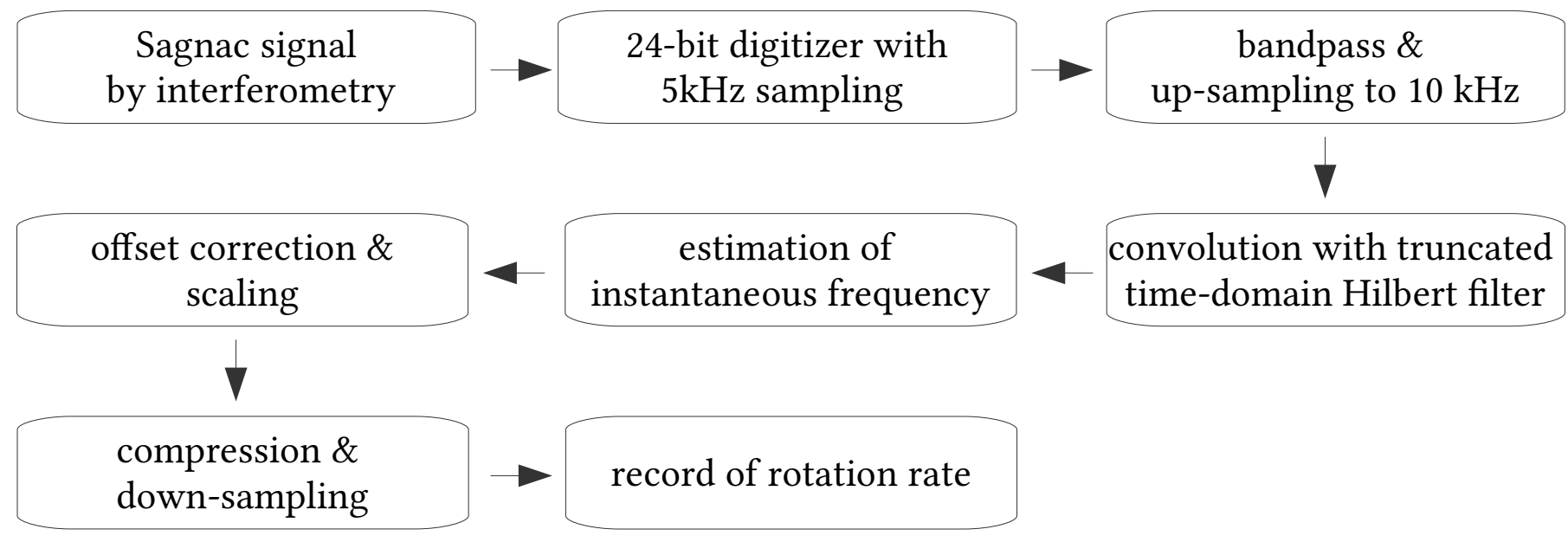

Figure 7: Schematic chart of the data acquisition and processing of ROMY. 


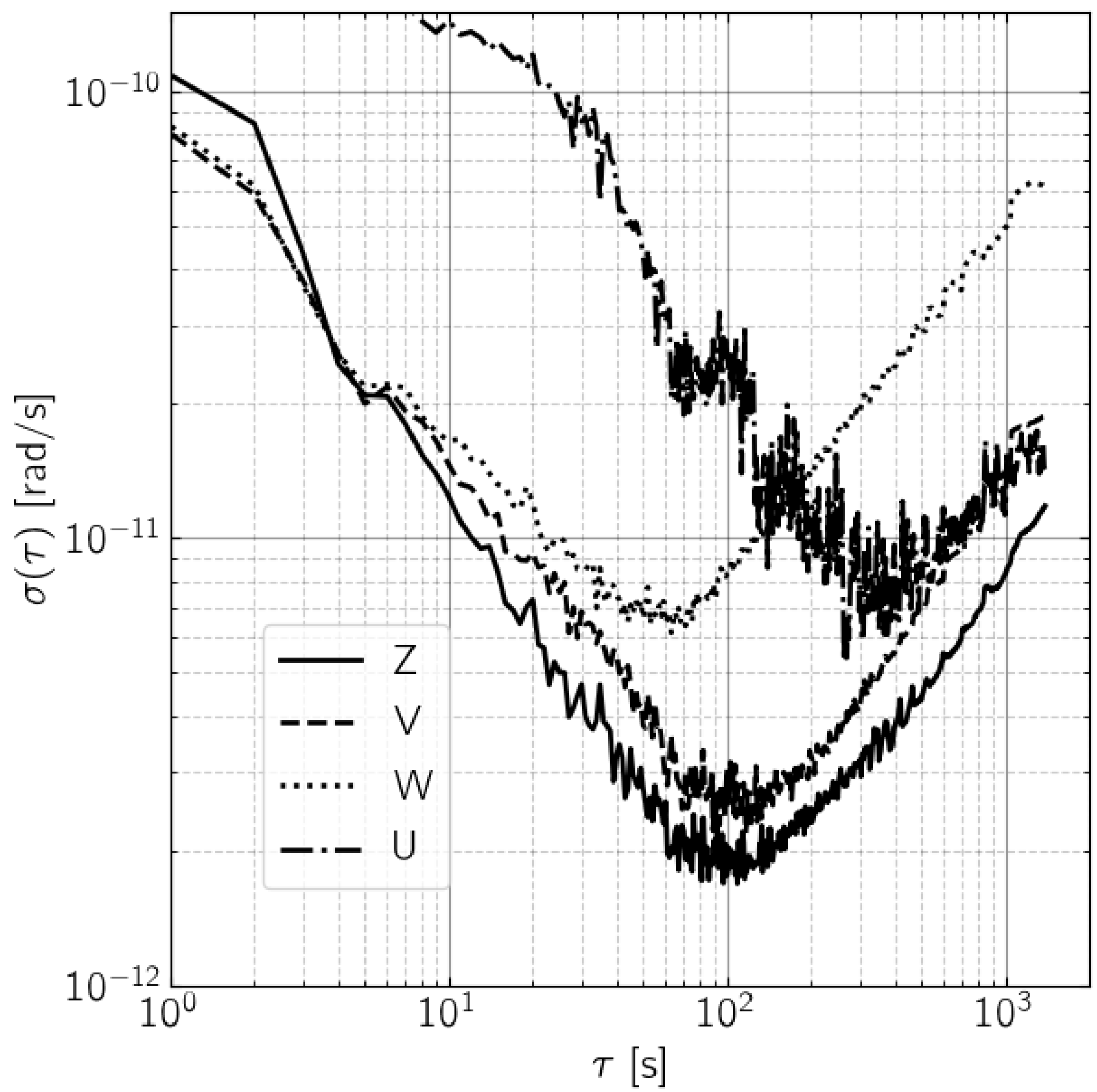

Figure 8: The Allan deviation $\sigma(\tau)$ of all four ROMY components. $\sigma(\tau)$ describes the sensor resolution after averaging over a time interval of length $\tau$. 

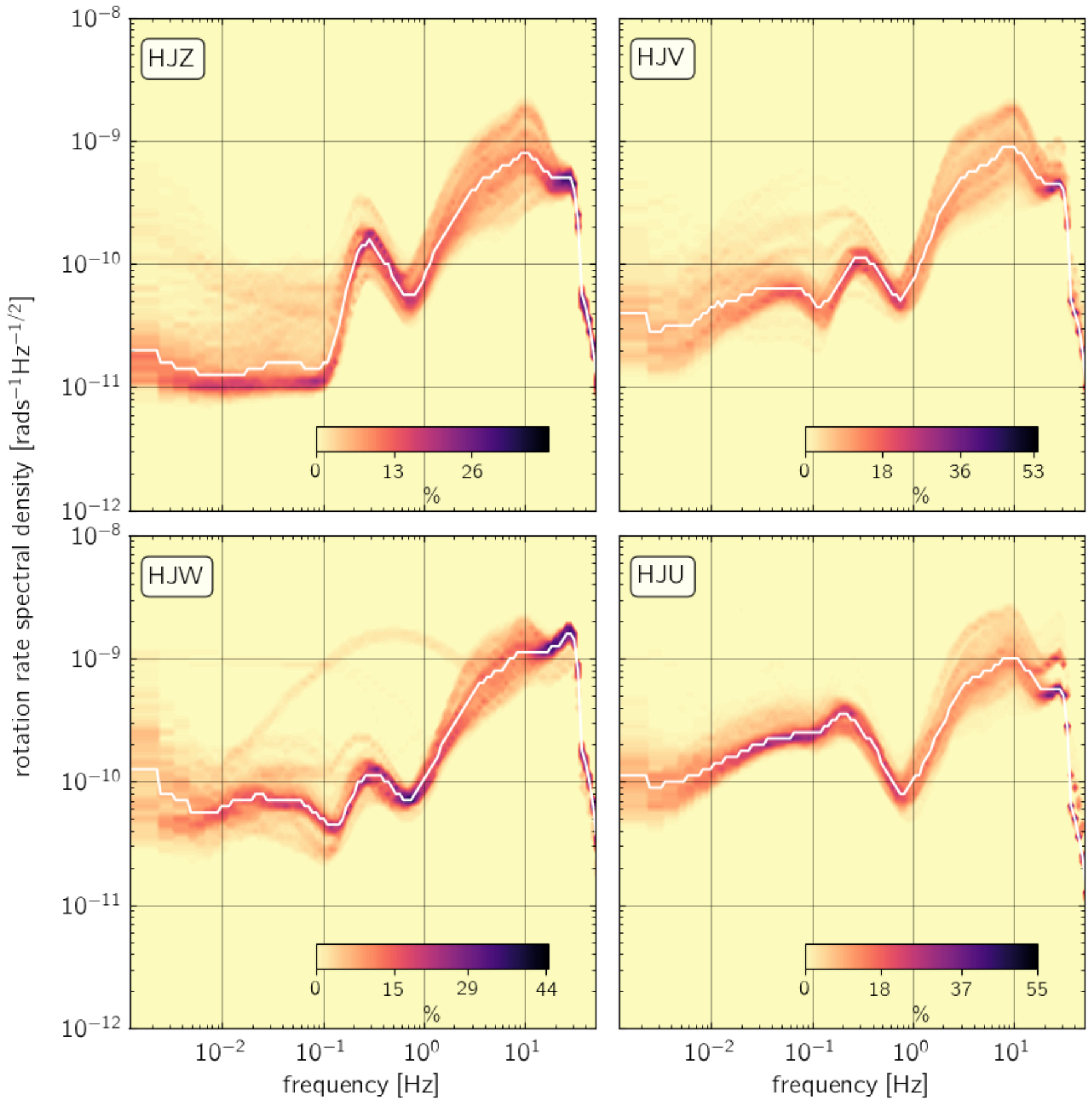

Figure 9: Probabilistic power spectral densities (PPSD) for all four ROMY components. The white line indicates the median of the distributions. 1 hour time windows with 50\% overlap were used to calculate power spectral densities. For each PPSD plot, we used around 30 continuous recordings each lasting 6 hours during periods of stable operation. In order to demonstrate station performance at low background noise levels, we exclude strong signals from nearby noise sources like farming machinery, with peak signal amplitudes exceeding $100 \mathrm{nrad} / \mathrm{s}$. 
(a)

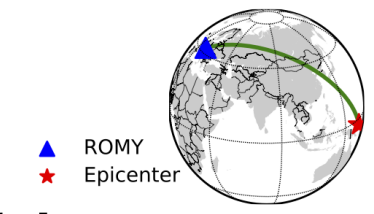

Papua New Guinea Earthquake

Time: 2019-05-14T12:58:25.939000Z (UTC)

Location: $46 \mathrm{~km}$ SSE of Namatanai, Papua New Guinea

Magnitude: $7.6 \mathrm{mww}$

Epicentral distance: $125.0^{\circ}$

Depth: $10.0 \mathrm{~km}$
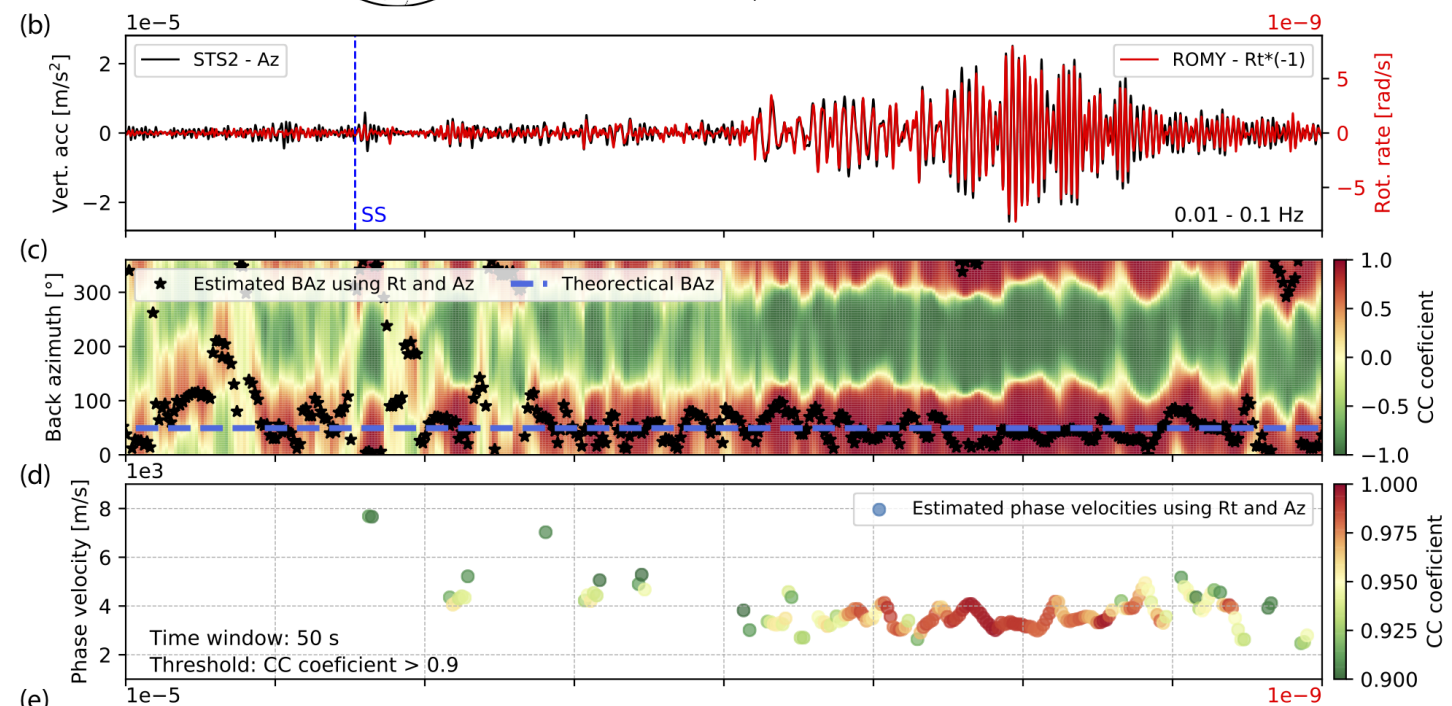

(e)
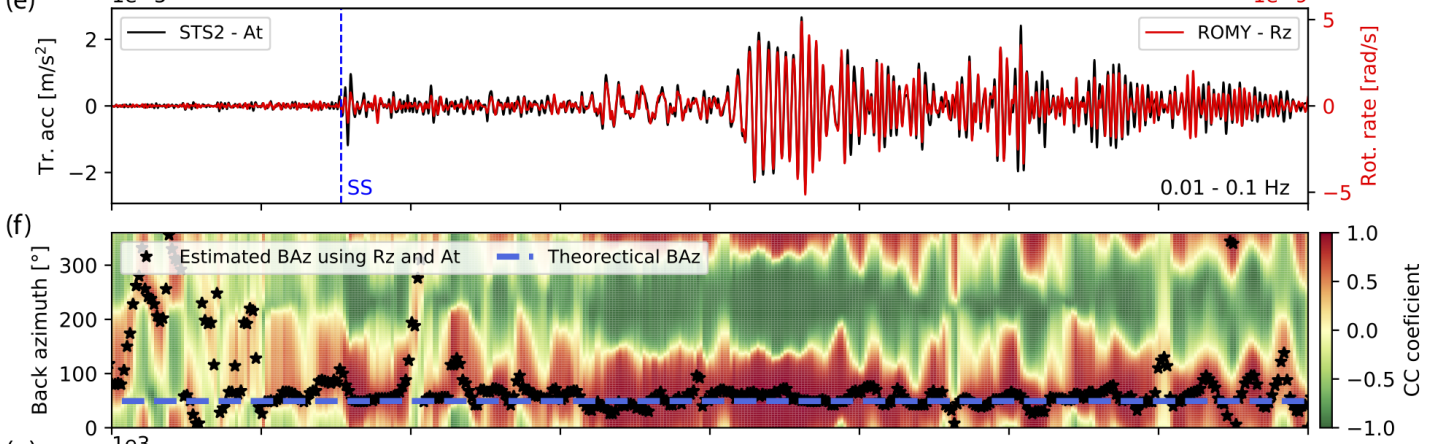

$$
\text { (9) }
$$



Figure 10: Observed translational and rotational motions for the M7.6 Papua New Guinea earthquake, May 14, 2019. (a) Earthquake information and schematic view of the great-circle-path through the epicentre and ROMY in Fürstenfeldbruck, Germany. (b) Superposition of the band-pass filtered (0.01-0.1 Hz) vertical acceleration (Az) and transverse rotational rate (Rt). (c) Estimated BAz for each $50 \mathrm{~s}$ sliding time window (black stars) using the cross-correlation method between Az and Rt. The color scale denotes the cross-correlation coefficient. The dashed blue line denotes the theoretical BAz. (d) Estimated phase velocities with cross-correlation (CC) coefficients higher than 0.9 for each sliding window using Az and Rt. (e-g) The same as (b-d), respectively, but for transverse acceleration (At) and vertical rotational rate (Rz), which focus on Love-type waves. 
(a)

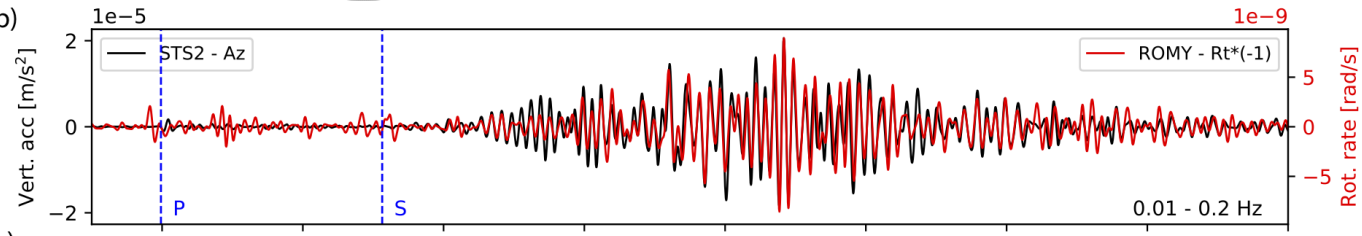

(c)

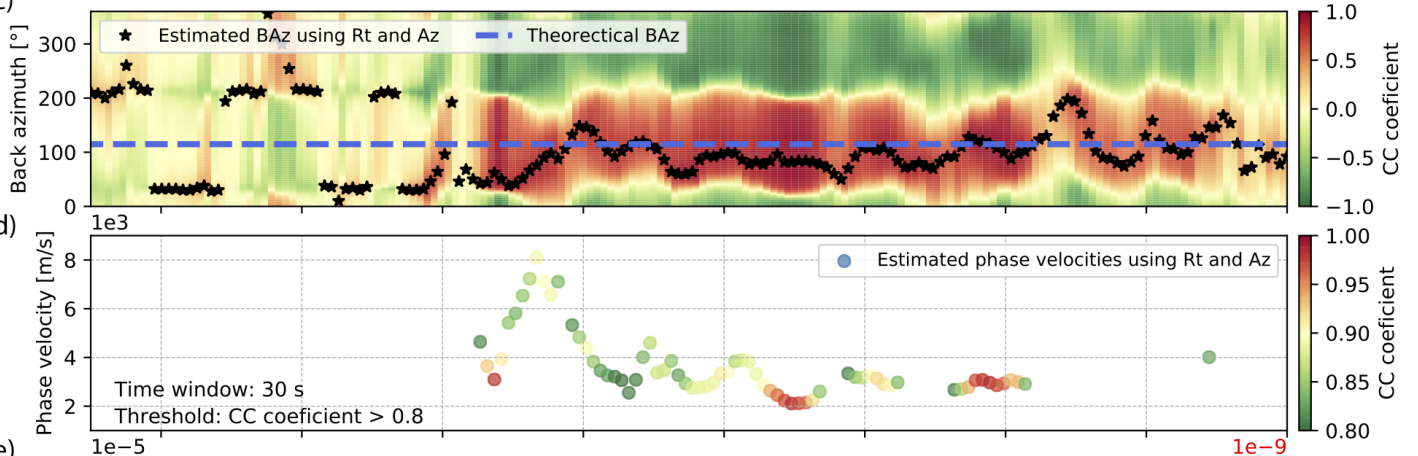

(e)

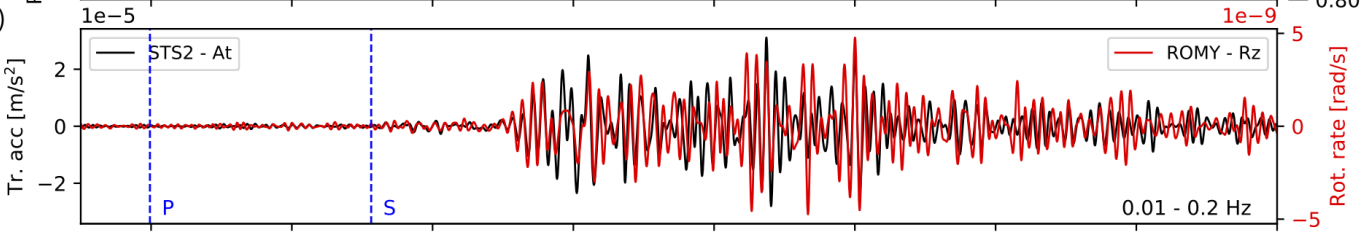

(f)

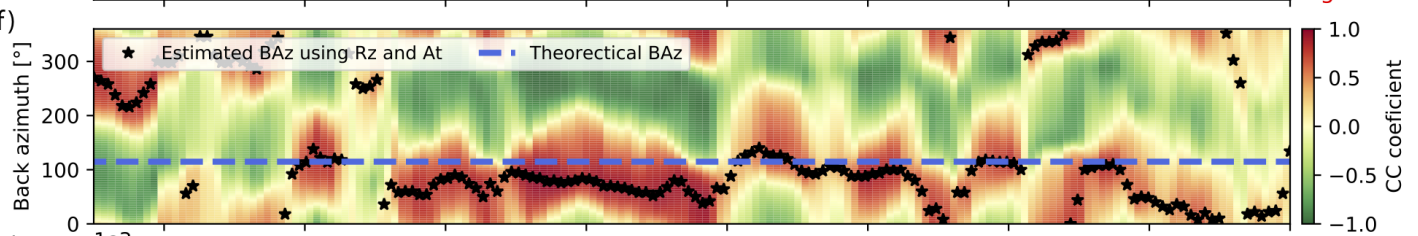

(g)

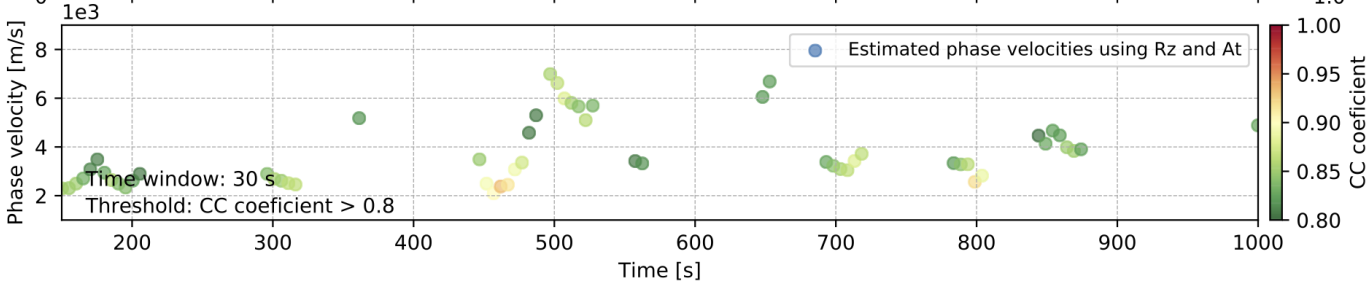

Figure 11: The same presentation as Fig. 10 but for the M5.7 Turkey earthquake, September 26, 2019. 
(a)

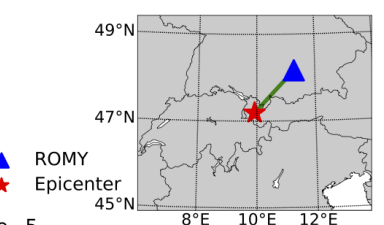

Austria Earthquake

Time: 2018-02-01T01:47:33.410000Z (UTC)

Location: $4 \mathrm{~km} \mathrm{~N}$ of Innerbraz, Austria

Magnitude: $3.8 \mathrm{ml}$

Epicentral distance: $1.3^{\circ}$

Depth: $5.0 \mathrm{~km}$

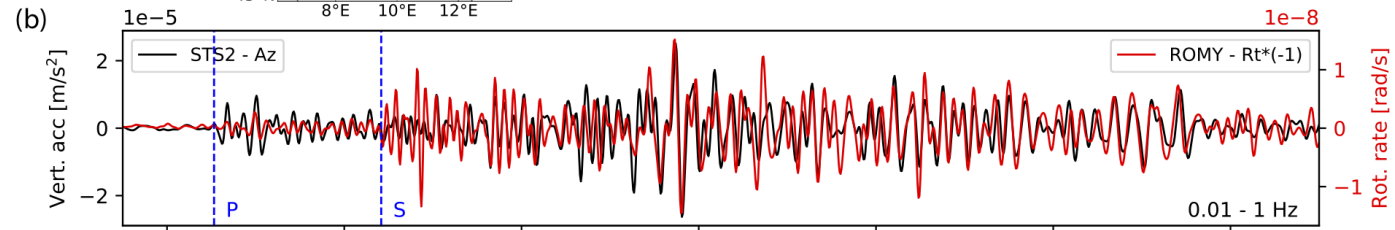

(c)

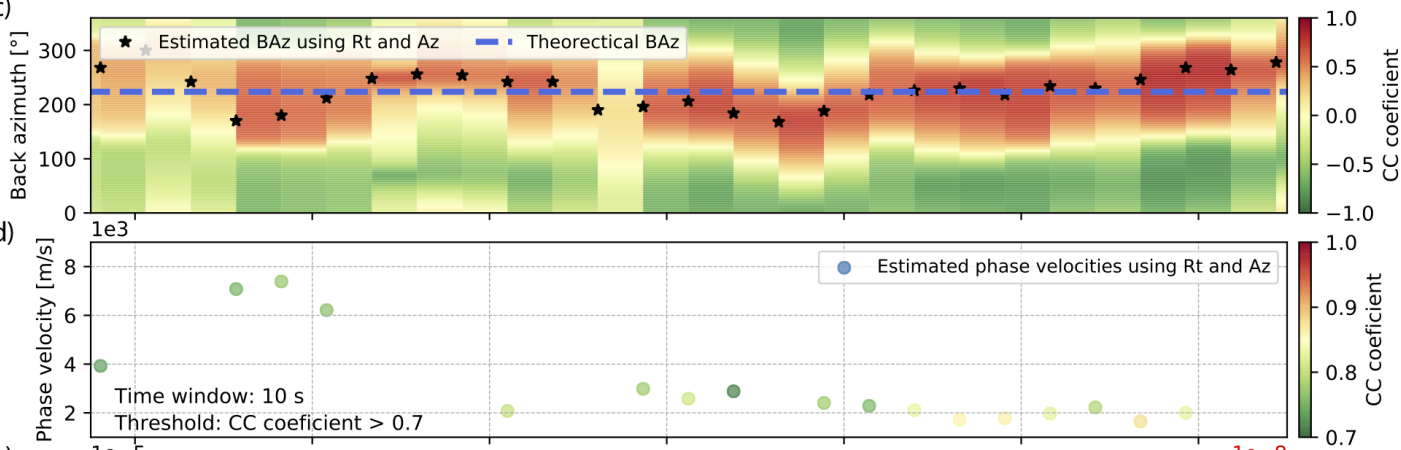

(e)
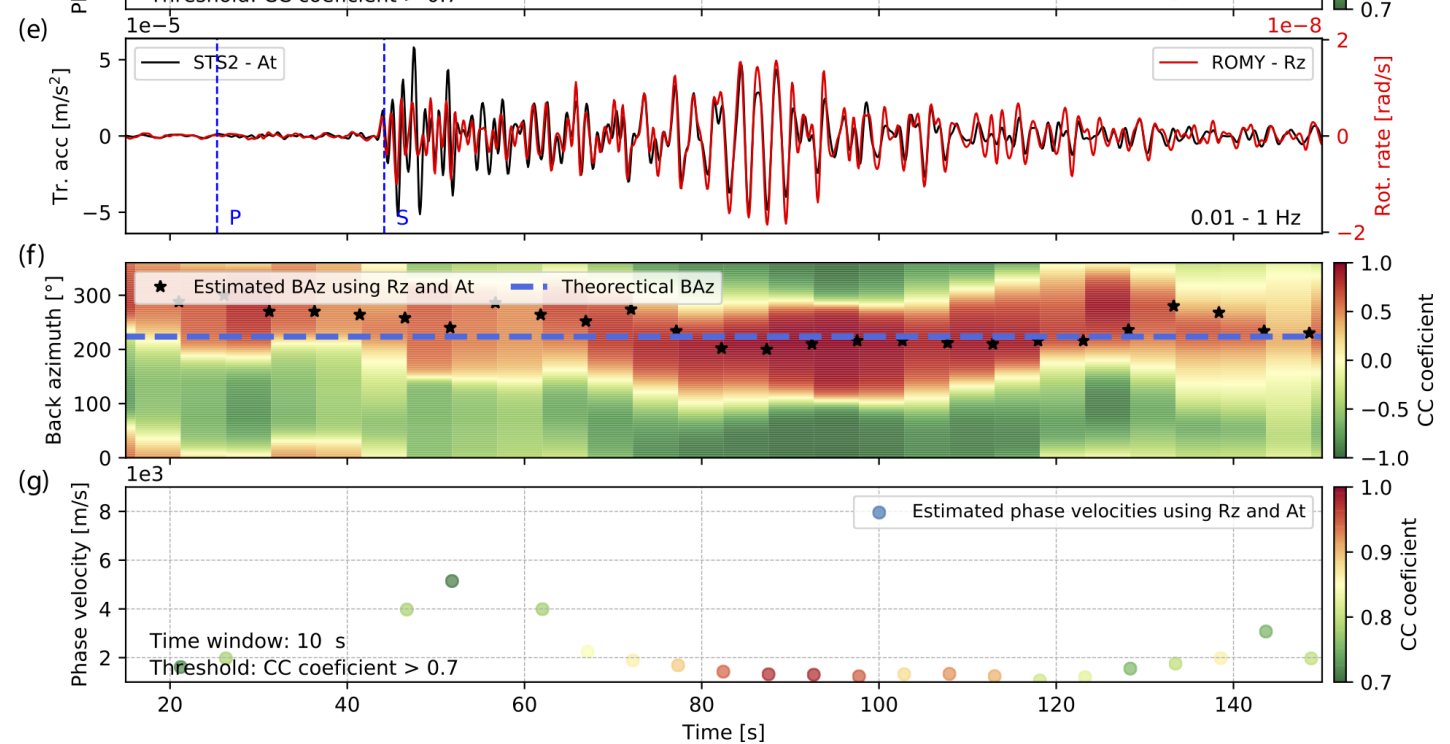

Figure 12: The same presentation as Fig. 10 but the M3.8 Austria earthquake, February 1, 2018. 

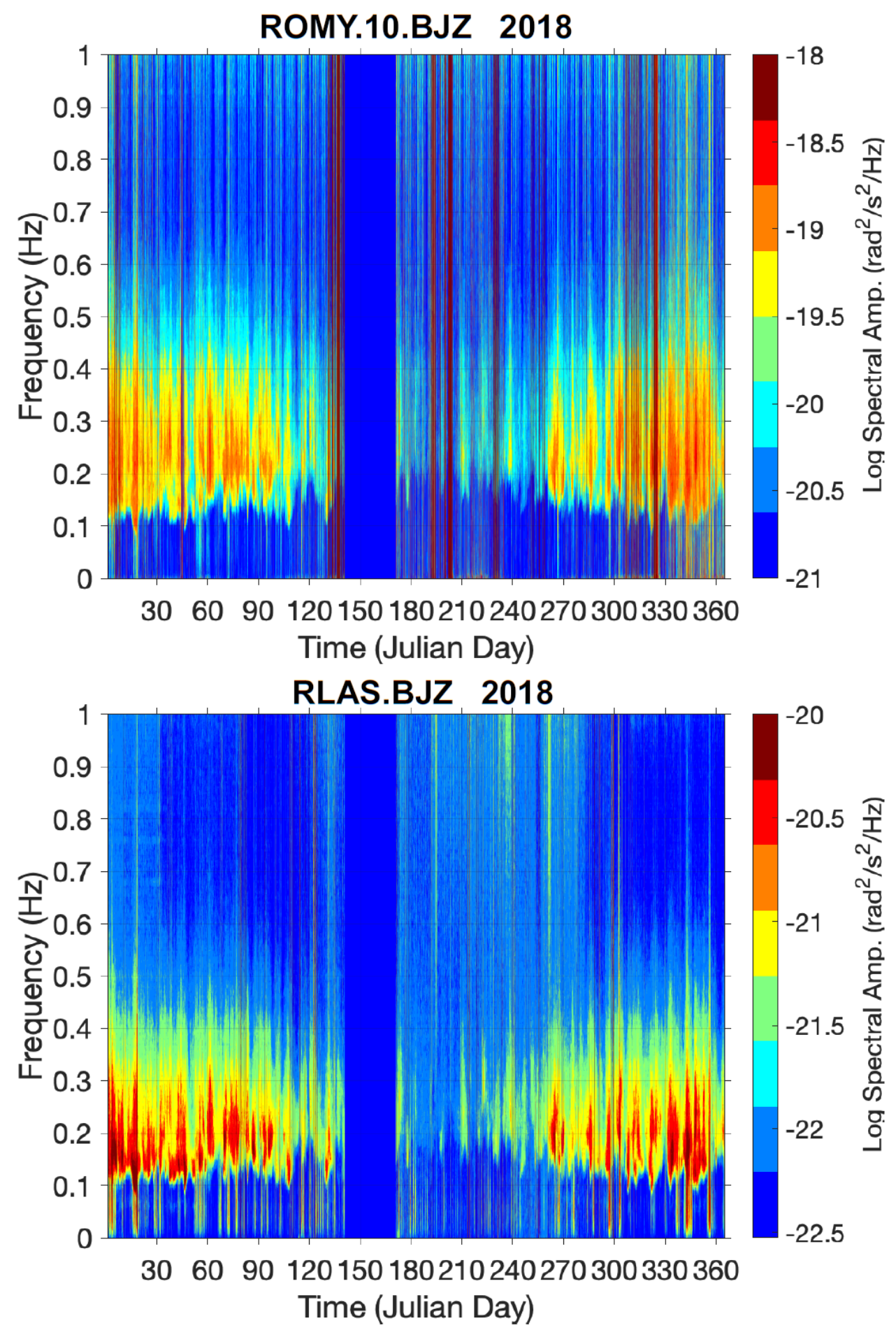

Figure 13: Seasonal variations of rotation noise. Top: Time-frequency plot of the rotation rate around the vertical axis of the Wettzell ring laser in 2018 in the interval $[0-1 H z]$. Bottom: Same for ROMY. In both cases we clearly see seasonal variations in the secondary microseismic band with periods in the range of 3-10 seconds. Note the different color scales, indicating the substantial amplitude difference (see text for details). 